\title{
Effects of Positive and Negative Expectations on Human Pain Perception Engage Separate But Interrelated and Dependently Regulated Cerebral Mechanisms
}

\author{
Yao-Wei Shih, ${ }^{1}$ Hsin-Yun Tsai, ${ }^{1}$ Feng-Sheng Lin, ${ }^{2}$ Yi-Hsuan Lin, ${ }^{1}$ Chun-Yen Chiang, ${ }^{1}$ Zheng-Liang Lu, ${ }^{3}$ \\ and $\odot$ Ming-Tsung Tseng ${ }^{1}$ \\ ${ }^{1}$ Graduate Institute of Brain and Mind Sciences, National Taiwan University College of Medicine, Taipei 10051, Taiwan, ${ }^{2}$ Department of Anesthesiology, \\ National Taiwan University Hospital, 10002, Taipei, Taiwan, and ${ }^{3}$ Department of Computer Science and Information Engineering, National Taiwan \\ University, Taipei, 10617, Taiwan
}

Expectations substantially influence pain perception, but the relationship between positive and negative expectations remains unclear. Recent evidence indicates that the integration between pain-related expectations and prediction errors is crucial for pain perception, which suggests that aversive prediction error-associated regions, such as the anterior insular cortex (aIC) and rostral anterior cingulate cortex (rACC), may play a pivotal role in expectation-induced pain modulation and help to delineate the relationship between positive and negative expectations. In a stimulus expectancy paradigm combining fMRI in healthy volunteers of both sexes, we found that, although positive and negative expectations respectively engaged the right aIC and right rACC to modulate pain, their associated activations and pain rating changes were significantly correlated. When positive and negative expectations modulated pain, the right aIC and rACC exhibited opposite coupling with periaqueductal gray (PAG) and the mismatch between actual and expected pain respectively modulated their coupling with PAG and thalamus across individuals. Participants' certainty about expectations predicted the extent of pain modulation, with positive expectations involving connectivity between aIC and hippocampus, a region regulating anxiety, and negative expectations engaging connectivity between rACC and lateral orbitofrontal cortex, a region reflecting outcome value and certainty. Interestingly, the strength of these certainty-related connectivities was also significantly associated between positive and negative expectations. These findings suggest that aversive prediction-error-related regions interact with pain-processing circuits to underlie stimulus expectancy effects on pain, with positive and negative expectations engaging dissociable but interrelated neural responses that are dependently regulated by individual certainty about expectations.

Key words: anterior cingulate cortex; expectation; functional magnetic resonance imaging; insula; pain; prediction error

\section{Significance Statement}

Positive and negative expectations substantially influence pain perception, but their relationship remains unclear. Using fMRI in a stimulus expectancy paradigm, we found that, although positive and negative expectations engaged separate brain regions encoding the mismatch between actual and expected pain and involved opposite functional connectivities with the descending pain modulatory system, they produced significantly correlated pain rating changes and brain activation. Moreover, participants' certainty about expectations predicted the magnitude of both types of pain modulation, with the underlying functional connectivities significantly correlated between positive and negative expectations. These findings advance current understanding about cognitive modulation of pain, suggesting that both types of pain modulation engage different aversive prediction error signals but are dependently regulated by individual certainty about expectations.

\section{Introduction}

The perception of pain in humans entails complex interactions between cognitive processes and nociceptive information, with the subjective experience of pain largely shaped by our expectations (Ploghaus et al., 2001; Koyama et al., 2005; Keltner et al., 2006; Atlas et al., 2010; Kong et al., 2013). While anticipating a 
pain stimulus, positive expectations (i.e., expectations of decreased pain) reduce pain perception (Koyama et al., 2005; Kong et al., 2013), whereas negative expectations (i.e., expectations of increased pain) have the opposite effect (Ploghaus et al., 2001; Keltner et al., 2006). Investigations on the relationship between positive and negative expectations to clarify whether the two cognitive constructs engage dependent or independent neural mechanisms are of crucial importance in understanding how stimulus expectancies influence pain experiences. Surprisingly, little attention to date has been paid to address this issue.

For both positive and negative expectations, how top-down expectations interact with bottom-up nociceptive inputs to modulate pain perception remains a fundamental question (Wiech, 2016). During pain anticipation, brain regions associated with pain processing, such as the thalamus, somatosensory cortex, and periaqueductal gray (PAG), have been found to become activated (Hsieh et al., 1999; Porro et al., 2002; Koyama et al., 2005; Fairhurst et al., 2007; Yoshida et al., 2013). During painful stimulation, an individual's certainty (i.e., the confidence in beliefs) and anxiety about expected pain have also been reported to predict the extent to which expectations modulate pain perception (Ploghaus et al., 2001; Brown et al., 2008; Gondo et al., 2012). Recently, it has been shown that the integration between expectations and prediction errors of pain (i.e., the mismatch between ascending nociceptive inputs and expectations), particularly the latter, is crucial for pain perception as well as relevant neurophysiological responses (Büchel et al., 2014; Geuter et al., 2017; Fazeli and Büchel, 2018). This observation raises the possibility that neural substrates encoding prediction errors for aversive events, such as the anterior insula (aIC) and rostral anterior cingulate cortex (rACC) (Seymour et al., 2004, 2005; Palminteri et al., 2012; Roy et al., 2014), may play a pivotal role in expectation-induced pain modulation. If this is the case, then one would predict that brain regions reflecting the mismatch between actual and expected pain would interact with pain-processing circuits to shape the perception of pain during painful stimulation. It is also plausible to anticipate that these brain regions would collaborate with the neural substrate associated with subjective certainty and/or anxiety about expectations, such as hippocampus and orbitofrontal cortex (OFC) (Rogers et al., 1999; Critchley et al., 2001; Ploghaus et al., 2001; Kong et al., 2008; Wiech et al., 2010; Freeman et al., 2015), to reflect the influence of these psychological factors on stimulus expectancy effects.

To investigate these hypotheses and determine whether these proposed mechanisms could delineate the relationship between positive and negative expectations, we used pain-predictive cues as stimulus expectancy manipulations to induce expectations about the magnitude of an upcoming pain stimulus. In a conditioning session, healthy volunteers learned the contingencies between cues and pain intensities. During the formal experiment, trials with correctly and incorrectly signaled medium pain stimulus allowed us to examine the impact of positive versus negative expectations on pain. By using fMRI, we first isolated brain areas encoding painful stimulus intensity and then examined the mechanisms by which brain regions reflecting the mismatch between actual and expected pain underlay expectation-induced

(Grant NHRI-EX106-10615NC), and the Imaging Center for Integrated Body, Mind and Culture Research at National Taiwan University.

The authors declare no competing financial interests.

Correspondence should be addressed to Ming-Tsung Tseng at mingtsungtseng@ntu.edu.tw.

https://doi.org/10.1523/JNEUROSCI.2154-18.2018

Copyright $\odot 2019$ the authors $\quad 0270-6474 / 19 / 391262-14 \$ 15.00 / 0$ pain modulation. We then elucidated how individual certainty and anxiety about predicted pain influenced stimulus expectancy effects on pain and examined the relationship between positive and negative expectations.

\section{Materials and Methods}

Participants. Twenty-eight healthy, right-handed volunteers who had never participated in pain studies before consented to participate in this study, which was approved by the Ethics Committee of National Taiwan University Hospital, Taipei, Taiwan. Five participants were eliminated from analyses: two reported minimal painful sensation to electrical stimulation, two explicitly stated they had no expectation when the cue appeared during scanning in the postscanning debriefing, and one was unable to differentiate between the three intensities of painful stimulation during the conditioning session. Therefore, data from 23 participants ( 10 females; age range: $20-32$ years; mean \pm SD: $24.1 \pm 3.4$ years) were analyzed.

Stimuli. Each painful electrocutaneous stimulus consisted of $100 \mathrm{~ms}$ monophasic rectangular pulses (pulse width: $2 \mathrm{~ms}$ at $20 \mathrm{~Hz}$ ) generated by a bipolar constant-current stimulator (DS5; Digitimer). Stimuli were delivered to the dorsum of the left hand via a pair of leads (LEAD108; Biopac Systems) and silver chloride surface electrodes (EL-508; Biopac Systems). All leads and electrodes were MRI compatible. All stimulus presentation and data collection were controlled by laptop computers equipped with Presentation software (Neurobehavioral Systems).

Behavioral session. Before the formal experiment, each participant took part in a behavioral session to determine three intensities of painful stimulation (designated as P2, P5, and P8) that elicited low, medium, and high pain [i.e., 2, 5, and 8 on a numerical rating scale from 0 (no pain) to 10 (unbearable pain)]. In the beginning, the detection threshold for just noticeable sensation was assessed by an ascending method of limits protocol (start: $0.1 \mathrm{~mA}$; step: $0.2 \mathrm{~mA}$; interstimulus interval: $10 \mathrm{~s}$ ). This was followed by two additional series of ascending stimuli (start: detection threshold; step: $0.5 \mathrm{~mA}$; interstimulus interval: $10 \mathrm{~s}$ ) that allowed a preliminary estimation of P2, P5, and P8. Subsequently, a calibration procedure was performed to ensure a more precise determination of P2, P5, and $\mathrm{P} 8$. In this procedure, $\mathrm{P} 2, \mathrm{P} 5$, and $\mathrm{P} 8$ obtained from the preceding method of limits procedures were delivered once and participants were instructed to rate their pain immediately after the application of each stimulus. For each intensity, if the rating was higher (or lower) than the target pain score (i.e., 2, 5, and 8, respectively), the intensity would be decreased (or increased) accordingly by $0.5 \mathrm{~mA}$. Based on this principle, the three intensities would then be adjusted to complete one round of calibration. In total, six rounds of calibration were administered.

Conditioning session. To establish stimulus expectancy effects on pain perception, a conditioning session identical in trial structure to the formal fMRI experiment (Fig. 1A) was administered. This session encompassed three trial types: a low-pain visual cue paired with a P2 stimulus (LL trial), a medium-pain visual cue paired with a P5 stimulus (MM trial), and a high-pain visual cue paired with a P8 stimulus (HH trial). Each trial started with a visual cue $(1.5 \mathrm{~s})$, which was followed by a $3-5 \mathrm{~s}$ (jitter) anticipation period. An electrical stimulus was then delivered and, after a $3.9 \mathrm{~s}$ poststimulus interval, participants had to rate subjective pain intensity on a $0-10$ visual analog scale (VAS; 0 : no pain; 10 : unbearable pain) within $3.5 \mathrm{~s}$. Participants were informed of the presence of three visual cues but unaware of the stimulus magnitude following each cue. They were instructed to establish the cue-stimulus relationships during this session. This session also served to familiarize participants with the experimental procedures during the formal imaging session. Six repetitions for each trial type were presented in randomized order.

Experimental design. The goal of the experimental manipulations in the formal experiment was threefold: (1) to maintain participants' beliefs about the expected pain intensity acquired during the conditioning session, (2) to induce expectations of decreased pain (i.e., positive expectations) and expectations of increased pain (i.e., negative expectations), and (3) to isolate brain regions tracking pain intensity without a significant bias (or at least a minimal bias) of specific expectations. To meet 
A

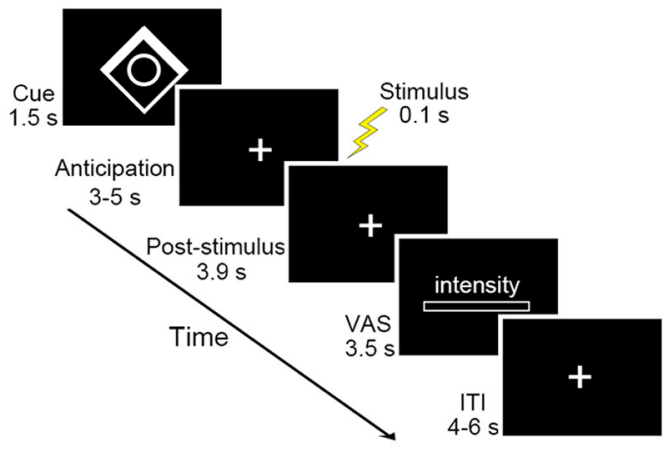

B

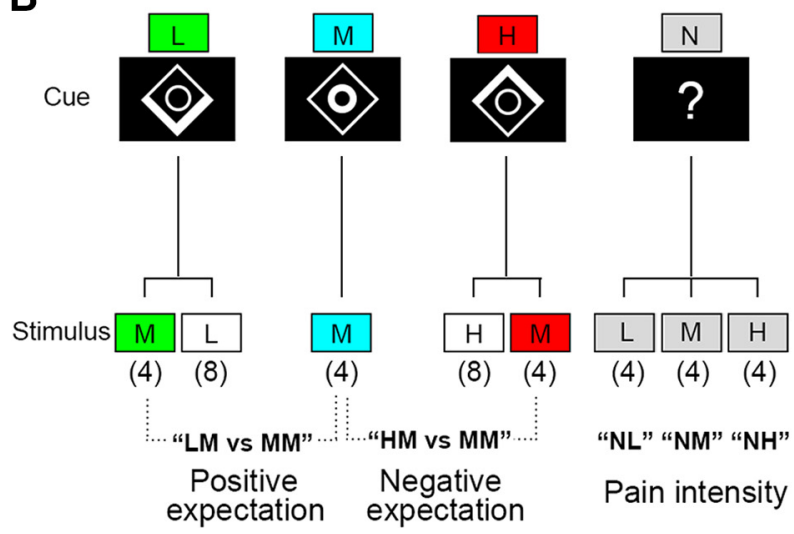

C

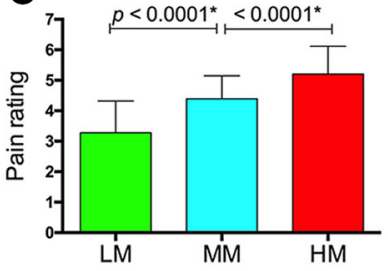

E

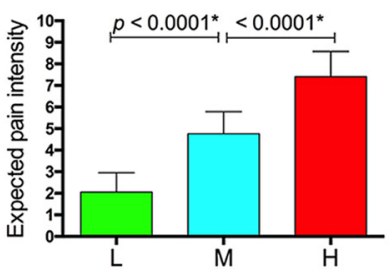

G

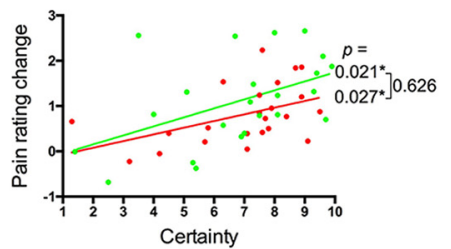

I

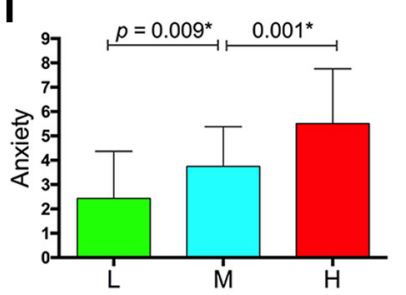

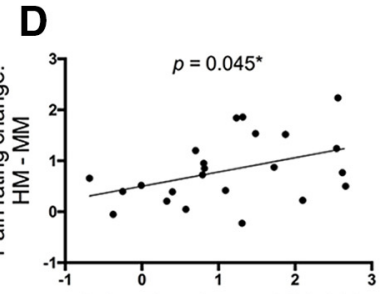

Pain rating change: $\mathrm{MM}-\mathrm{LM}$

$\mathbf{F}$

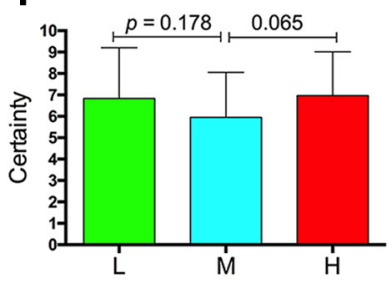

H
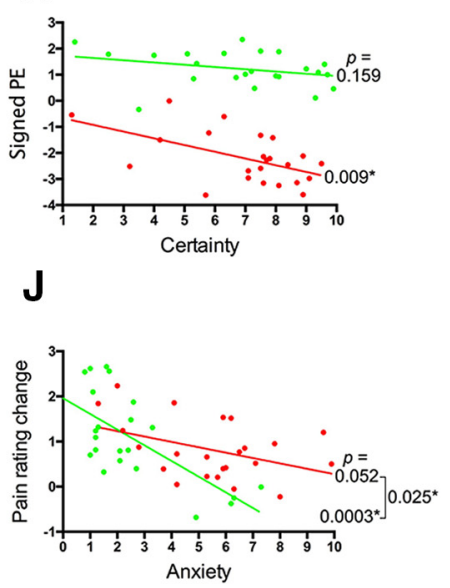

Figure 1. Experimental design and behavioral results. $A$, Each trial was initiated with a $1.5 \mathrm{~s}$ pain-predictive visual cue (a high-pain cue in this example). After a $3-5 \mathrm{~s}$ anticipation period, a $0.1 \mathrm{~s}$ electrical pain stimulus was applied to the dorsum of the left hand and, after a $3.9 \mathrm{~s}$ delay, participants reported subjective pain intensity on a VAS within $3.5 \mathrm{~s}$. The intertrial interval was $4-6 \mathrm{~s}$. B, Cue-stimulus contingencies. The conditioned visual cue was a compound image consisting of a square and a circle. The lower and upper part of the square brightened to constitute the low- and high-pain cue, respectively. Brightening of the inner circle denoted the medium-pain cue. On trials without a conditioned cue, the visual cue consisted of a question mark. The numbers in parentheses indicate the number of repetitions of each trial type in a single scanning session (totally 40 trials per session). C, Compared with a medium-pain cue and during the receipt of an identical medium pain stimulation, a low- and high-pain cue significantly reduced and increased subjective pain intensity, respectively (both $p<0.0001)$. $\boldsymbol{D}$, The change in pain rating caused by a low-pain cue was positively correlated with that caused by a high-pain cue $(p=0.045)$. , The low- and high-pain cue respectively elicited a significantly lower and higher expected pain intensity compared with that provoked by a medium-pain cue (both $p<0.0001$ ). $\boldsymbol{F}$, The cue-elicited subjective certainty about expected pain was not significantly different among the three cues ( $p>0.065$ for all pairwise comparisons). G, For both low- and high-pain cues, subjective certainty ratings were positively correlated with the extent of pain rating changes ( $p=0.021$ for the low-pain cue and $p=0.027$ for the high-pain cue), with no significant difference between both correlations $(p=0.626)$. $\boldsymbol{H}$, The subjective certainty elicited by a high-pain cue exhibited a significant negative correlation with the signed prediction error (PE) of pain (i.e., reported pain intensity - expected pain intensity) engendered in the HM condition $(p=0.009)$. The correlation associated with a low-pain cue was not significant $(p=0.159)$. $I$, The anxiety level elicited by a low- and high-pain cue was significantly lower $(p=0.009)$ and higher $(p=0.001)$ than that provoked by a medium-pain cue. $J$, For a low-pain cue, the level of provoked anxiety was negatively correlated with the corresponding change in pain rating $(p=0.0003)$. These relationships did not reach statistical significance for a high-pain cue $(p=0.052$ ) and the correlation related to a low-pain cue was significantly stronger than that associated with a high-pain cue $(p=0.025)$. Error bars in $\boldsymbol{C}, \boldsymbol{E}, \boldsymbol{F}$, and $I$ represent $S D$ s. ${ }^{*} p<0.05$.

these requirements sequentially, the present cue-based paradigm encompassed three types of trials: (1) three correctly signaled trials identical to those used in the conditioning session (i.e., LL, MM, and HH trials), (2) two incorrectly signaled trials with expectations for decreased pain (i.e., LM trial, a low-pain cue followed by a P5 stimulus) and expectations for increased pain (i.e., HM trial, a high-pain cue followed by a P5 stimulus), and (3) three trials without a conditioned visual cue (i.e., NL, NM, and $\mathrm{NH}$ trials, an unlearned visual cue followed by a P2, P5, or P8 stimulus, respectively; Fig. 1B). Different from a previous study (Atlas et al., 2010), the inclusion of the trial with correctly signaled medium pain stimulus (i.e., MM trial) in our paradigm allowed us to compare the effects between positive and negative expectations. To maintain participants' beliefs about the cue-stimulus contingencies, the number of HH and LL trials (each with eight repetitions per session) doubled the number of HM and LM trials (each with four repetitions per session). To elucidate the effect of positive (i.e., LM trial) and negative (i.e., HM trial) expectations on pain, the total number of LM, HM, and MM trials was equal (each with four repetitions per session). To isolate brain areas encoding pain intensity, each session included the same proportion of the NL, NM, and NH trials (each with four repetitions per session). Because clear effects of stimulus expectancy on pain had already been observed without any verbal instruction about cue-stimulus contingencies in a behavioral pilot study, we did not inform participants about contingencies throughout the entire experimental session, which minimized the potential confounding effects related to verbal information retrieval. In total, the fMRI session consisted of three scanning runs. Each run contained 40 trials (Fig. $1 B$ ), with each trial type presented in randomized order. As described above, participants were requested to report subjective pain intensity in each trial using a VAS presented on the monitor during scanning. Trials were interleaved with intertrial intervals of 4-6 s (jitter). 
Postscanning rating and behavioral data analysis. Immediately after scanning, participants were presented with the same pain-predictive cues on a computer screen and were asked to use a $0-10$ VAS to report their average anticipated pain intensity during the formal experiment ("When you saw this cue, how much pain did you expect?" 0: no pain; 10: unbearable pain). To assess whether positive and negative expectations were successfully provoked and to evaluate the mismatch between actual and expected pain engendered in each trial types, the anticipated pain intensity elicited by the low-, medium-, and high-pain cue was compared with the reported pain rating during correctly cued conditions (i.e., LL, MM, and $\mathrm{HH}$ conditions), as well as the reported pain rating during the NL, $\mathrm{NM}$, and $\mathrm{NH}$ conditions, in which the received pain was not biased (or at least minimally biased) by anticipation of specific pain intensity. Because participants' certainty and anxiety about expected pain have been shown to affect the impact of expectations on pain experiences (Ploghaus et al., 2001; Brown et al., 2008; Gondo et al., 2012), they were also asked to use VASs to rate their average level of certainty ("Regarding this cue, how certain were you about the relationship between this cue and the intensity of the subsequent painful stimulus?" 0: very uncertain; 10: very certain) and anxiety ("When you saw this cue, how anxious were you about the following pain?" 0: not anxious; 10 : very anxious) about their expectations during the formal experiment. To investigate how subjective certainty and anxiety modulated stimulus expectancy effects on pain, these postscanning behavioral data were correlated with the extent of pain rating changes as well as the difference between expected and reported pain intensities (i.e., prediction errors of pain) during LM trials (for positive expectations) and HM trials (for negative expectations) across individuals.

Statistical analysis. Statistical tests were performed with SPSS and GraphPad Prism software. Paired $t$ tests were used to compare reported pain intensity during the fMRI experiment and psychological measures after scanning. One-way repeated-measures ANOVA was used to compare pain ratings and the effect of expectations on pain across the three scanning sessions. Pearson's correlation test was used to examine the linear relationship between two continuous variables. Analysis of covariance was used to test whether slopes of two linear regression lines were significantly different from each other.

$f M R I$ data acquisition. Whole-brain fMRI data were collected using a 3-tesla Magnetom Prisma system equipped with a 64-channel head coil (Siemens). Blood oxygen-level dependent (BOLD) responses were recorded using a gradient-echo $\mathrm{T} 2^{\star}$-weighted echoplanar imaging (EPI) sequence with the following parameters: 37 contiguous axial slices; $\mathrm{TR}=$ $2000 \mathrm{~ms} ; \mathrm{TE}=30 \mathrm{~ms}$; flip angle $=90^{\circ} ; \mathrm{FOV}=224 \times 224 \mathrm{~mm}$; a GRAPPA acceleration factor of 2; slice thickness $=3.9 \mathrm{~mm}$; acquisition matrix $=64 \times 64$; voxel size $=3.5 \times 3.5 \times 3.9 \mathrm{~mm}$. The initial four EPI volumes were discarded to allow for the stabilization of the BOLD signal. To correct for magnetic field inhomogeneity (Hutton et al., 2002), a map of the static magnetic field using the same slice geometry as EPI images was acquired using a double gradient-echo sequence $\left(\mathrm{TR}=600 \mathrm{~ms}, \mathrm{TE}_{1}=\right.$ $\left.10.00, \mathrm{TE}_{2}=12.46 \mathrm{~ms}\right)$. For registration purposes, a high-resolution T1weighted magnetization-prepared rapid-acquisition gradient echo image (voxel size $0.88 \times 0.88 \times 0.89 \mathrm{~mm}$ ) and a structural T2-weighted scan coplanar with the functional images but with higher in-plane resolution $(256 \times 256)$ were additionally acquired.

Imaging data analysis. Imaging data were analyzed using SPM8 (Wellcome Department of Imaging Neuroscience, London, UK). All EPI volumes were unwarped using field maps and subsequently corrected for motion artifacts and differences in the acquisition timing of each slice. The resulting mean EPI image was coregistered with the participant's T2-weighted structural image, which in turn was aligned with the T1weighted image. To improve intersubject registration, the coregistered T1-weighted images were then segmented to gray matter, white matter, and CSF according to the ICBM space template for East Asia brains to generate a study-specific template and individual flow fields using the Diffeomorphic Anatomical Registration Through Exponentiated Lie algebra (DARTEL) toolbox. The flow fields contained the deformation parameters to this study-specific template for each participant and were used to normalize each participant's realigned and resliced functional images to the standard Montreal Neurological Institute (MNI) space, with spatial resolution of $2 \times 2 \times 2 \mathrm{~mm}$. Finally, data were smoothed using a Gaussian kernel with a full-width-half-maximum of $6 \mathrm{~mm}$. All participants' T1-weighted images were averaged for overlay of statistical parametric maps. The time series was high-pass filtered to $1 / 128 \mathrm{~Hz}$ to remove low-frequency noise.

The general linear model (GLM) of SPM8 was used to conduct fMRI data analyses (Friston et al., 1995). For each participant, a first-level model was constructed as a sequence of events convolved with the canonical hemodynamic response function. The current study focused on the stimulation period, during which we set 8 regressors (duration: $0.1 \mathrm{~s}$ ) to encompass BOLD signals for the LM, LL, MM, HH, HM, NL, NM, and $\mathrm{NH}$ trials (Fig. 1B). The anticipation period contained 4 regressors for the $\mathrm{H}, \mathrm{M}, \mathrm{L}$, and $\mathrm{N}$ cues (duration: 3-5 s). Other regressors of no interest included the period of cue (all conditions collapsed to 1 regressor; duration: $1.5 \mathrm{~s}$ ), the rating period (all conditions collapsed to 1 regressor; duration: $3.5 \mathrm{~s}$ ), and the six head-motion parameters estimated during the realignment procedure (Friston et al., 1997). Each participant's firstlevel $t$-contrasts were then entered into second-level GLM analyses to investigate contrasts of interest (Holmes and Friston, 1998). In all activation-related group contrasts analyzed in the current study, age and gender were included as covariates of no interest to regress out their potential confounding effects on pain-related activations (Greenspan et al., 2007; Tseng et al., 2013).

We first confirmed activations within pain-processing brain regions by analyzing the BOLD signals during NL, NM, and NH trials (i.e., contrasts "NL," "NM," and "NH"; Fig. 1B) using whole-brain analyses. To assess brain regions encoding pain intensity, we analyzed a linear trend contrast of $(-1,0,1)$ (i.e., "NL $<\mathrm{NM}<\mathrm{NH}^{\prime}$ ) with a whole-brain analysis as well as a small-volume correction (SVC) analysis in the right thalamus, the main brain region receiving ascending nociceptive inputs (Craig, 2003). Second, anticipation-related activations were identified by examining the BOLD signal during the anticipation period. Another whole-brain analysis was conducted to determine whether painanticipation-associated activation pattern was consistent with previous neuroimaging studies. Third, to investigate whether stimulus expectancy effects on pain involved brain areas coding for the mismatch between actual and expected pain intensity, we compared medium pain-related activations between correctly and incorrectly signaled trials (i.e., contrasts "LM > MM" and "HM > MM") and performed SVCs in brain areas implicated in processing prediction error of pain, including the aIC, rACC, and PAG (described below) (Seymour et al., 2004, 2005; Roy et al., 2014). Given that the ventral striatum (VS) has been well characterized to show reward prediction error coding (Schultz, 1998, 2016), we performed separate SVCs in this region to clarify whether reward prediction-error-related regions also underlay stimulus expectancy effects on pain. Note that activity within these prediction-error-related regions has been reported to reflect both positive and negative prediction errors for aversive stimuli (Seymour et al., 2005; Delgado et al., 2008). Because we did not ask participants to report expected pain intensity before the receipt of pain stimulation in each trial (Schenk et al., 2017), we could not perform trialwise analyses to isolate neural prediction error signals nor could we determine whether prediction errors updated expectations. Alternatively, we compared the response profile in identified brain areas associated with expectation-induced pain modulation (i.e., right aIC and right rACC) across different trial conditions to determine whether they represented prediction errors of pain in our experiment (Ploghaus et al., 2000). Note that, as described above, participants were asked to report their average anticipated pain intensity after scanning. Therefore, the term "prediction error" in our experiment refers to the average mismatch between actual and expected outcomes engendered in each trial type.

Psychophysiological interaction (PPI) analysis. A PPI analysis investigates context-dependent differences in functional connectivity (i.e., the temporal correlation in BOLD signals) with a seed region, which allows an assessment of how the functional interaction between different brain areas changes with different experimental conditions (Friston et al., 1997). Because our results revealed that activations in the right aIC and rACC were respectively associated with positive and negative expectancy effects on pain, we used the generalized PPI toolbox in 
SPM (http://www.nitrc.org/projects/gppi) (McLaren et al., 2012) and used the right aIC and right rACC as the seed to run separate PPI analyses (Friston et al., 1997) and test the following two hypotheses. First, we investigated whether expectations biased pain through affecting the interaction between the right aIC or rACC and the key structures in ascending and descending pain pathways. In this analysis, we performed SVCs in the right thalamus to assess the interaction with the ascending pain pathway and in the PAG, the control center for descending pain modulation (Tracey and Mantyh, 2007), to determine the connectivity with the descending pain pathway. Second, we investigated whether the modulation of individual certainty and anxiety about pain expectations was reflected by the interaction between the right aIC or rACC and brain areas associated with outcome certainty and anxiety to pain, specifically the OFC and hippocampus (Rogers et al., 1999; Critchley et al., 2001; Ploghaus et al., 2001; Kong et al., 2008; Wiech et al., 2010; Freeman et al., 2015). In this analysis, SVCs were conducted in these hypothesized regions.

To investigate whether functional connectivities associated with stimulus expectancy effects on pain were modulated by the mismatch between actual and expected pain across subjects, we examined the relationship between the strength of functional connectivity and the individual prediction error of pain. Analogous to previous research (Schenk et al., 2017), the prediction error of pain associated with positive expectations was calculated by subtracting the expected pain intensity elicited by a low-pain cue from the reported pain rating in LM trials and the prediction error of pain related to negative expectations was estimated by subtracting the expected pain intensity elicited by a high-pain cue from the reported pain rating in HM trials. Because we found that the response profile in the right aIC might reflect positive prediction errors of pain (see Results for details), a simple linear regression analysis was performed to determine whether prediction errors related to the intensity of pain covaried with the strength of aIC-PAG coupling extracted from the PAG ROI. This analysis was then complemented by a grouplevel regression analysis in SPM using the individual prediction error of pain in the LM condition as a covariate with SVCs in the PAG. For negative expectations, we performed a multiple linear regression analysis using the rACC-thalamus coupling strength extracted from the ROI for the right thalamus as dependent variable and pain prediction errors and pain rating during the $\mathrm{NH}$ condition as independent variables because we found that the response pattern of the right rACC appeared to consist of an absolute prediction error component and a nociceptive processing component (see Results for details). This analysis was also complemented by a group-level multiple linear regression analysis in SPM using the individual prediction error of pain in the $\mathrm{HM}$ condition and pain rating during the $\mathrm{NH}$ (as well as $\mathrm{HH}$ ) condition as covariates with a SVC analysis in the right thalamus. In these regression analyses, both signed and absolute prediction errors of pain were analyzed.

For a PPI analysis, we first extracted the deconvolved time courses from the right aIC and right rACC ROIs (described below) for each participant to create the physiological regressor. Subsequently, the extracted time courses were multiplied with the different experimental conditions (i.e., psychological regressor; "LM," "HM," and "MM") to constitute the PPI term. Each participant's PPI maps were then entered into a second-level random-effects group analysis.

ROI definition. To test our hypotheses, we independently defined key brain areas associated with ascending nociceptive inputs (thalamus), descending pain modulation (PAG), aversive prediction errors (aIC, rACC, and $P A G$ ), and subjective certainty and anxiety to pain (hippocampus and OFC) as ROIs. Considering pain specificity, the ROI for the right thalamus was built as a 4 -mm-radius sphere centered at MNI coordinates $(6,-20,10)$ as reported in a meta-analysis on pain-related activations (Duerden and Albanese, 2013). Given that the aim of the current study was to clarify the role of pain-related prediction error signals, we focused on the right ventrolateral part of the PAG because the ventrolateral part of the PAG has particularly been implicated in aversive learning (McNally et al., 2011; Herry and Johansen, 2014) and the right PAG, which receives most nociceptive information from the left hand (i.e., our pain stimulation site) (Yezierski, 1988) and the top-down modulation from the cortical areas (i.e., expectations) (Mantyh, 1982; Floyd et al.,
2000), is in good position to compute the difference between ascending nociceptive inputs and expectations (i.e., prediction errors of pain) to subsequently modulate pain in bilateral dorsal horns (Morgan et al., 2008). The mask of this PAG subregion was generated according to connectivity-based segmentation of the PAG in humans (Ezra et al., 2015; Faull and Pattinson, 2017). For the aIC and rACC, we defined 5-mm-radius spherical masks for the bilateral aIC [centered at $( \pm 36,28$, $6)]$ and rACC [centered at $(2,34,18)$ and $(-642-12)]$ based on the coordinates associated with aversive prediction errors reported in a recent meta-analysis (Garrison et al., 2013). The aversive prediction-errorrelated ROIs (i.e., aIC, rACC, and PAG) were combined into a single ROI mask to correct for the number of different ROIs when we performed SVCs to isolate brain regions related to expectation-induced pain modulation. The ROI mask for the VS encompassed two 5-mm-radius spherical masks centered at $( \pm 18,8,-14)$ (Garrison et al., 2013). For the ROI associated with subjective certainty about expectations, we created spherical ROIs for the orbitofrontal cortex [ $10 \mathrm{~mm}$ radius; centered at $( \pm 36,44,-4)]$ according to prior work on pain modulation by negative expectations (Freeman et al., 2015). With regard to pain-related anxiety, we used the automated anatomical labeling ROI library (TzourioMazoyer et al., 2002) to define the ROI for the bilateral hippocampus. For both the orbitofrontal cortex and the hippocampus, bilateral ROIs were combined into a single mask for SVCs.

In the present study, we adopted a nonparametric, permutation-based approach (SnPM13; http://warwick.ac.uk/snpm) (Nichols and Holmes, 2002) with 5000 permutations and without variance smoothing to control for false-positives due to multiple testing in group-level whole-brain analyses (Eklund et al., 2016). Results were considered significant based on a cluster-level familywise error (FWE) rate of $p<0.05$. For ROI analyses, SVCs were performed in SPM using a voxelwise threshold of $p<0.05$, FWE corrected. To accurately depict SVC results, only suprathreshold voxels were illustrated.

\section{Results}

\section{Behavioral results}

In trials without a conditioned visual cue (i.e., NL, NM, and $\mathrm{NH}$ trials), low-, medium-, and high-pain stimulation produced graded subjective pain ratings (mean \pm SD: $1.53 \pm 0.71,4.63 \pm$ 0.95 , and $6.64 \pm 0.57$, respectively; $p<0.0001$ for all pairwise comparisons). For each of the three stimulus intensities, the average pain ratings were not significantly different among the three scanning sessions (all $p>0.324$ ). Within each session, participants' pain intensity ratings for the first and the last painful stimulation were not significantly different for each of the three stimulus intensities (all $p>0.139$ ), suggesting that no significant habituation or sensitization toward the repetitive painful stimuli occurred throughout the experiment.

As expected, while receiving a medium pain stimulation, a low- and high-pain cue significantly reduced and increased participants' subjective pain ratings compared with the mediumpain cue, respectively (mean $\pm \mathrm{SD}: 3.28 \pm 1.04,4.39 \pm 0.75$, and $5.21 \pm 0.91$ for the low-, medium-, and high-pain cue, respectively; both $p<0.0001$; Fig. $1 C$ ). The effect of expectations on pain ratings was not significantly different among the three sessions $\left(F_{(2,44)}=0.906, p=0.397\right.$ for positive expectations; $F_{(2,44)}=1.367, p=0.265$ for negative expectations), which indicates that the stimulus expectancy effects remained constant across the entire scanning session. Interestingly, there was a significant positive correlation in the magnitude of pain rating changes between positive and negative expectations across individuals $(r=0.422, p=0.045$; Fig. $1 D)$.

For postscanning cue-related ratings, participants reported a gradual increase in average predicted pain intensities for the three pain-predictive cues $(2.06 \pm 0.89,4.76 \pm 1.03$, and $7.41 \pm 1.16$ for the low-, medium-, and high-pain cue, respectively; $p<$ 0.0001 for all pairwise comparisons; Fig. $1 E$ ), suggesting that par- 
Table 1. Whole-brain analyses during anticipation and stimulation periods

\begin{tabular}{|c|c|c|c|c|c|c|c|c|c|c|c|c|c|c|c|c|}
\hline \multirow[b]{3}{*}{ Area } & \multirow[b]{3}{*}{ Side } & \multicolumn{3}{|l|}{ Anticipation } & \multicolumn{12}{|l|}{ Stimulation } \\
\hline & & \multicolumn{3}{|l|}{ H } & \multicolumn{3}{|l|}{ NL } & \multicolumn{3}{|l|}{ NM } & \multicolumn{3}{|l|}{$\mathrm{NH}$} & \multicolumn{3}{|l|}{$\mathrm{NL}<\mathrm{NM}<\mathrm{NH}$} \\
\hline & & $x / y / z$ & $t$ & Cluster no. & $x / y / z$ & $t$ & Cluster no. & $x / y / z$ & $t$ & Cluster no. & $x / y / z$ & $t$ & Cluster no. & $x / y / z$ & $t$ & Cluster no. \\
\hline \multirow[t]{2}{*}{$\overline{\text { SII }}$} & $R$ & - & - & - & $40 /-14 / 18$ & 7.53 & 1 (2259) & $40 /-14 / 20$ & 9.27 & $1(5350)$ & $52 /-12 / 16$ & 9.60 & 1 1(6712) & $40 /-16 / 18$ & 6.32 & 1 (727) \\
\hline & L & - & - & - & $-40 /-8 / 14$ & 6.34 & $2(540)$ & $-38 /-18 / 18$ & 9.29 & $2(3558)$ & $-60 /-20 / 20$ & 8.70 & $2(5782)$ & $-44 /-16 / 20$ & 6.36 & $2(128)$ \\
\hline \multirow[t]{2}{*}{ SI } & $\mathrm{R}$ & - & - & - & - & - & - & - & - & - & $62 /-8 / 16$ & 9.00 & 1 & $42 /-18 / 46$ & 5.66 & $3(255)$ \\
\hline & L & - & - & - & $-58 /-18 / 22$ & 7.72 & $3(444)$ & $-60 /-18 / 22$ & 8.64 & 2 & $-58 /-18 / 22$ & 8.32 & 2 & & & \\
\hline \multirow[t]{2}{*}{ alc } & $\mathrm{R}$ & - & - & - & $30 / 14 /-18$ & 6.22 & 1 & $30 / 24 /-4$ & 7.35 & 1 & $38 / 16 / 2$ & 8.62 & 1 & & & \\
\hline & L & - & - & - & $-36 / 4 /-12$ & 6.81 & 2 & $-34 / 16 / 2$ & 7.29 & 2 & $-36 / 22 / 0$ & 10.38 & 2 & & & \\
\hline \multirow[t]{2}{*}{$\mathrm{plc}$} & R & - & - & - & $40 /-2 /-10$ & 7.29 & 1 & $44 /-14 / 12$ & 8.47 & 1 & $40 /-10 / 16$ & 12.47 & 1 & $42 /-14 /-2$ & 6.31 & 1 \\
\hline & L & - & - & - & $-34 /-14 / 18$ & 7.53 & 2 & $-40 /-10 / 8$ & 7.90 & 2 & $-38 /-18 / 16$ & 9.65 & 2 & & & \\
\hline \multirow[t]{2}{*}{ ACC } & R & - & - & - & - & - & - & - & - & - & $2 / 36 / 12$ & 7.39 & $3(2266)$ & & & \\
\hline & L & - & - & - & - & - & - & $-12 / 32 / 26$ & 4.76 & $3(1510)$ & $-8 / 26 / 26$ & 5.51 & 3 & & & \\
\hline \multirow[t]{2}{*}{ MCC } & R & $8 / 8 / 38$ & 6.66 & 1 (143) & - & - & - & 12/20/44 & 6.40 & 3 & $6 /-22 / 30$ & 7.09 & $4(405)$ & & & \\
\hline & L & - & - & - & $-8 / 16 / 42$ & 4.40 & $4(323)$ & $-12 / 24 / 34$ & 4.52 & 3 & $-4 /-14 / 32$ & 5.09 & 4 & & & \\
\hline \multirow[t]{2}{*}{ PCC } & R & - & - & - & - & - & - & $8 /-36 / 26$ & 6.29 & $4(313)$ & $8 /-38 / 26$ & 4.44 & 4 & & & \\
\hline & L & - & - & - & - & - & - & $-4 /-38 / 24$ & 4.13 & 4 & - & - & - & & & \\
\hline \multirow[t]{2}{*}{ Thalamus } & $\mathrm{R}$ & - & - & - & - & - & - & - & - & - & $14 /-14 / 6$ & 5.50 & $5(277)$ & & & \\
\hline & L & - & - & - & - & - & - & - & - & - & $-10 /-18 / 6$ & 5.74 & $6(358)$ & & & \\
\hline \multirow[t]{2}{*}{ SFG } & $\mathrm{R}$ & $14 /-4 / 72$ & 5.56 & $2(722)$ & - & - & - & - & - & - & - & - & - & & & \\
\hline & L & - & - & - & - & - & - & $-26 / 0 / 50$ & 6.87 & 3 & $-22 / 4 / 50$ & 5.49 & $7(221)$ & & & \\
\hline \multirow[t]{2}{*}{ MFG } & $\mathrm{R}$ & - & - & - & - & - & - & $38 / 14 / 38$ & 5.03 & 3 & $46 / 32 / 22$ & 4.25 & $8(318)$ & & & \\
\hline & L & - & - & - & - & - & - & $-36 / 4 / 58$ & 4.26 & 3 & $-42 / 40 / 24$ & 4.19 & 2 & & & \\
\hline \multirow[t]{2}{*}{ IFG } & $\mathrm{R}$ & $56 / 8 / 10$ & 5.12 & $3(199)$ & - & - & - & $34 / 18 / 26$ & 5.99 & 1 & 40/14/26 & 5.28 & 8 & & & \\
\hline & L & - & - & - & - & - & - & $-42 / 10 / 28$ & 7.42 & 2 & $-32 / 30 / 0$ & 10.51 & 2 & & & \\
\hline SMA & $\mathrm{R}$ & $8 /-6 / 56$ & 5.89 & 2 & - & - & - & - & - & - & 0/10/60 & 6.29 & 3 & & & \\
\hline & L & $-6 /-8 / 66$ & 5.50 & 2 & $-10 / 16 / 48$ & 6.62 & 4 & $-6 / 12 / 54$ & 8.64 & 3 & $-6 / 16 / 48$ & 8.26 & 3 & & & \\
\hline PMA & R & $40 /-6 / 62$ & 5.13 & 2 & - & - & - & - & - & - & - & - & - & & & \\
\hline & L & $62 / 8 / 24$ & 4.74 & 3 & - & - & - & $-32 /-4 / 50$ & 6.02 & 3 & $-34 /-4 / 50$ & 6.78 & 7 & & & \\
\hline PPC & $\mathrm{R}$ & - & - & - & $64 /-18 / 26$ & 7.24 & 1 & $52 /-34 / 28$ & 3.66 & 1 & $50 /-32 / 26$ & 5.47 & 1 & & & \\
\hline & L & - & - & - & $-60 /-30 / 40$ & 4.64 & 3 & $-34 /-56 / 36$ & 5.32 & $5(160)$ & $-56 /-40 / 38$ & 4.06 & 2 & & & \\
\hline Precuneus & R & - & - & - & - & - & - & $16 /-60 / 24$ & 6.43 & $6(2089)$ & $14 /-62 / 22$ & 7.03 & $9(2040)$ & & & \\
\hline & L & - & - & - & - & - & - & $-14 /-64 / 34$ & 7.30 & 6 & $-8 /-64 / 44$ & 5.25 & $10(303)$ & & & \\
\hline STG & $\mathrm{R}$ & - & - & - & - & - & - & $42 /-22 / 0$ & 7.44 & 1 & $50 /-24 / 10$ & 4.69 & 1 & & & \\
\hline & L & - & - & - & - & - & - & $-42 /-14 /-4$ & 6.73 & 2 & $-40 /-6 /-8$ & 11.64 & 2 & & & \\
\hline ve & $\mathrm{R}$ & - & - & - & - & - & - & $6 /-72 / 8$ & 5.04 & 6 & $10 /-88 / 18$ & 5.86 & 9 & & & \\
\hline & L & - & - & - & - & - & - & $-12 /-72 / 2$ & 5.88 & 6 & $-14 /-64 / 26$ & 7.05 & 10 & & & \\
\hline PHG & $\mathrm{R}$ & - & - & - & - & - & - & - & - & - & $24 /-40 /-4$ & 5.68 & 9 & & & \\
\hline & L & - & - & - & - & - & - & - & - & - & $-18 /-40 /-4$ & 4.58 & 6 & & & \\
\hline Cerebellum & R & - & - & - & - & - & - & $32 /-76 /-50$ & 5.29 & $7(311)$ & $0 /-66 /-26$ & 5.32 & $11(394)$ & $2 /-52 /-36$ & 4.64 & $4(215)$ \\
\hline & L & - & - & - & - & - & - & - & - & - & $-12 /-66 /-34$ & 6.19 & 11 & $-4 /-64 /-34$ & 5.32 & 4 \\
\hline
\end{tabular}

Shown are activated brain areas of whole-brain analyses using SnPM (familywise error correction at a cluster-level of $p<0.05$ ) during the anticipation of high pain stimulation (contrast " $\mathrm{H}^{\text {") }}$ ) and during the stimulation period (contrasts "NL," "NM," "NH," and "NL $<\mathrm{NM}<\mathrm{NH}$ "). Activated clusters (with sizes in voxels in parentheses) are numbered and activation foci corresponding to different anatomical regions within the clusters are given as MNI coordinates (in millimeters). $\mathrm{ACC}$, Anterior cingulate cortex; alC, anterior insular cortex; IFG, inferior frontal gyrus; L, left; $\mathrm{MCC}$, middle cingulate cortex; $\mathrm{MFG}$, middle frontal gyrus; $\mathrm{PCC}$, posterior cingulate cortex; $\mathrm{PHG}$, parahippocampal gyrus; $\mathrm{plC}$, posterior insular cortex; PMA, premotor area; PPC, posterior parietal cortex; R, right; SFG, superior frontal gyrus; SI, primary somatosensory cortex; SII, secondary somatosensory cortex; SMA, supplementary motor area; STG, superior temporal gyrus; VC, visual cortex.

ticipants' beliefs about cue-stimulus contingencies remained held at the end of the experiment. Crucially, compared with the reported pain intensity during the NM condition, the mediumpain cue was associated with a comparable expected pain intensity $(p=0.642)$, whereas the low- and high-pain cue evoked a significantly lower and higher expected pain intensity (both $p<$ $0.0001)$. Note that the expected pain intensity evoked by the lowand high-pain cue was significantly higher than the reported pain intensity during the NL $(p=0.010)$ and $\mathrm{NH}(p=0.005)$ conditions, respectively. Comparisons between anticipated pain intensities with the reported pain rating during the LL, MM, and $\mathrm{HH}$ conditions yielded similar results. These findings indicate that positive and negative expectations were successfully provoked in our experimental paradigm. They also suggest no prediction errors of pain in the MM condition, positive prediction errors of pain in the LM condition, or negative prediction errors of pain in the HM, LL, and $\mathrm{HH}$ conditions.

For cue-elicited subjective certainty ratings, there was no significant difference among the three pain-predictive cues $(6.84 \pm$ $2.34,5.96 \pm 2.10$, and $6.97 \pm 2.04$ for the low-, medium-, and high-pain cue, respectively; $p>0.065$ for all pairwise comparisons; Fig. $1 F$ ). This is very important for an unbiased comparison between positive expectation- and negative expectation-induced pain modulation given the level of certainty potentially confounds brain responses related to expectancy effects (Yoshida et al., 2013; Seidel et al., 2015). Low- and high-pain cue-related certainty ratings, respectively, exhibited a significant positive correlation with pain rating changes associated with positive (i.e., MM-LM; $r=0.477, p=0.021$ ) and negative (i.e., HM-MM; $r=$ $0.462 ; p=0.027)$ expectations across subjects, with no significant difference between both correlations $\left(F_{(1,42)}=0.241, p=0.626\right.$; Fig. $1 G)$. Participants with a higher level of certainty regarding a high-pain cue exhibited a larger difference between expected pain intensity and reported pain intensity (i.e., a smaller signed prediction error of pain) during the HM condition $(r=-0.532, p=$ $0.009)$, but the relationship associated with the low-pain cue failed to reach statistical significant $(r=-0.304, p=0.159$; Fig. $1 H)$.

With regard to anxiety ratings, the low- and high-pain cue provoked a significantly lower $(p=0.009)$ and higher $(p=$ $0.001)$ level of anxiety compared with the medium-pain cue, respectively $(2.44 \pm 1.94,3.75 \pm 1.63$, and $5.51 \pm 2.25$ for the low-, medium-, and high-pain cue, respectively; Fig. $1 I$ ). The change in pain ratings associated with positive expectations (i.e., MM-LM) was negatively correlated with the level of anxiety evoked by a low-pain cue $(r=-0.682, p=0.0003)$, but the correlation associated with negative expectations was not significant $(r=$ $-0.411, p=0.052$; Fig. $1 J)$. The correlation associated with positive expectations was significantly stronger than that with negative expectations $\left(F_{(1,42)}=5.386, p=0.025\right)$. Indeed, the 

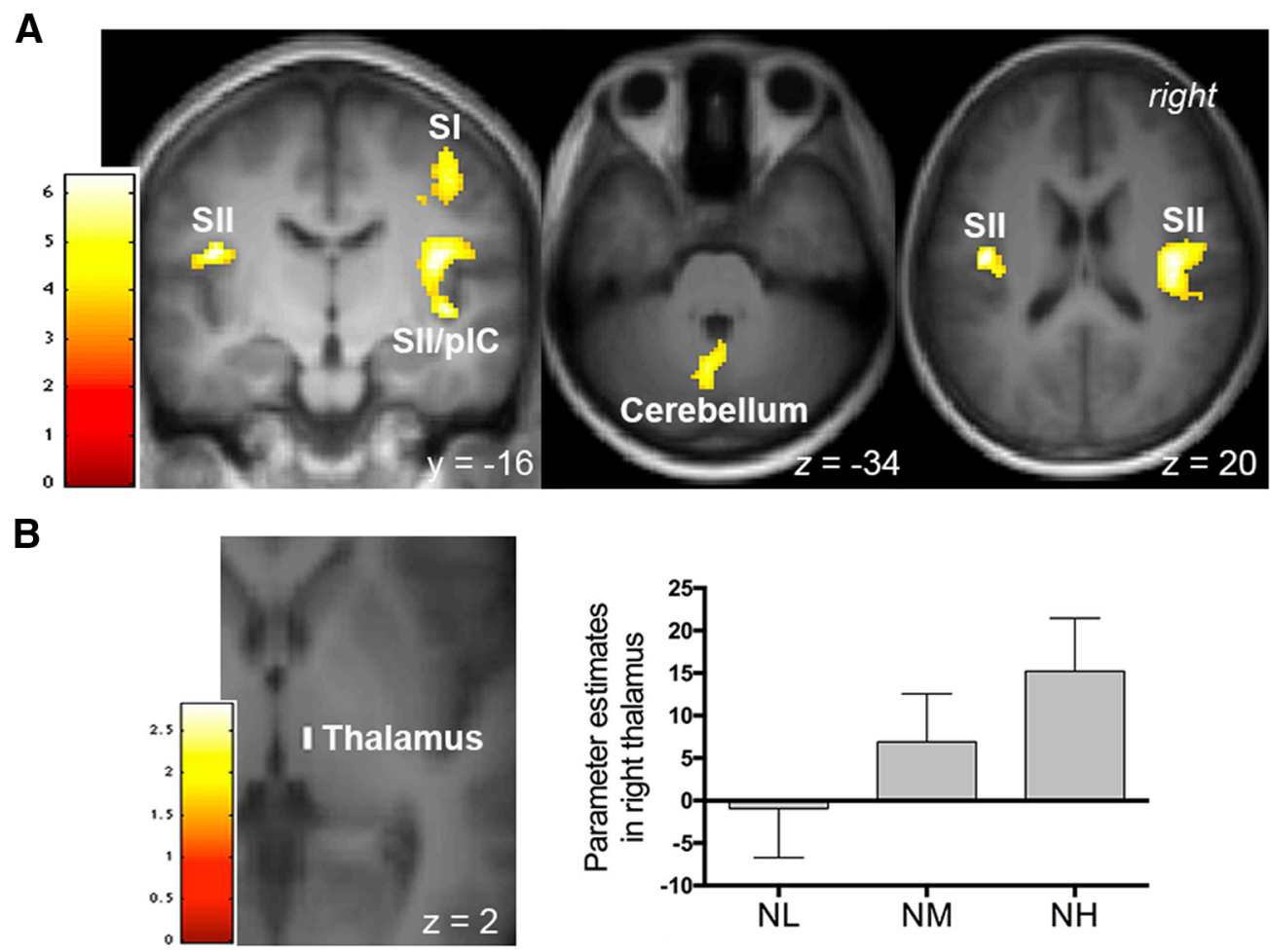

Figure 2. Brain regions reflecting intensity coding of pain. Activations were identified from the contrast "NL $<N M<N H$." $A$, A whole-brain analysis revealed significant activations in the right primary somatosensory cortex (SI), bilateral SII, right posterior insular cortex (pIC), and cerebellum. $\boldsymbol{B}$, Activation within the right thalamus survived small-volume corrections ( $p<0.05$, FWE corrected). Bar graphs depict parameter estimates extracted from the suprathreshold cluster. Error bars represent SEM. $\boldsymbol{A}, \boldsymbol{B}$, Activations were overlapped on an average structural image. The bar on the left shows the range of $t$ scores for SPM 8.

low-pain cue-provoked anxiety rating was also inversely correlated with its associated certainty rating (both with MM-LM; $r=$ $-0.652, p=0.0007$ ), but this relationship for the high-pain cue did not reach statistical significance (both with HM-MM; $r=$ $-0.194, p=0.376$ ). Together, these findings suggest that subjective certainty about expected pain comparably influenced the extent of positive expectation- and negative expectation-induced pain modulation, with anxiety selectively affecting positive expectation-induced pain modulation.

\section{Pain- and pain anticipation-related activation}

For a validity check, we first performed whole-brain analyses to examine pain- and pain anticipation-related activations. Consistent with previous reports, a conjunction analysis testing for the activation common to the three painful stimulus intensities (i.e., contrasts "NL," "NM," and "NH") revealed significant responses within typical pain-processing brain regions (Apkarian et al., 2005; Duerden and Albanese, 2013), including the bilateral secondary somatosensory cortex (SII), bilateral insula, and left middle cingulate cortex, as well as the left supplementary motor area and bilateral posterior parietal cortex (whole-brain correction; Table 1). The whole-brain analysis on the contrast "NL $<\mathrm{NM}<\mathrm{NH}$ " showed significant activations within the right primary somatosensory cortex, bilateral SII, right posterior IC, and bilateral cerebellum (Fig. $2 A$, Table 1). Consistent with the role of the thalamus in receiving ascending nociceptive inputs (Craig, 2003), activations in the right thalamus also increased linearly with increasing pain intensity (peak MNI coordinates $x / y / z=$ $8 /-22 / 2, t_{(1,20)}=2.82, p=0.041$ and $x / y / z=8 /-18 /-2, t_{(1,20)}$ $=2.78, p=0.044$; SVCs, FWE-corrected; Fig. $2 B$ ).
As for pain anticipation-related activations, a whole-brain analysis during the anticipation of a high pain revealed activations in the prefrontal and motor-related regions, including the right middle cingulate cortex, right superior frontal gyrus, right inferior frontal gyrus, bilateral supplementary motor area, and bilateral premotor area (Table 1). Consistent with previous neuroimaging studies (Ploghaus et al., 2001; Koyama et al., 2005; Keltner et al., 2006), for trials with high pain stimulation, a conjunction analysis revealed that anticipation-related (i.e., contrast " $\mathrm{H}$ ") and pain-related (i.e., contrast "NH") activations overlapped in the right middle cingulate cortex, right inferior frontal gyrus, and bilateral supplementary motor area. Whole-brain analyses during the anticipation of low and medium pain stimulation did not reveal any suprathreshold activations.

\section{Activation associated with stimulus expectancy effects on pain}

Having confirmed that pain- and pain anticipation-related activation patterns were consistent with previous studies, we then explored the neural substrates underlying stimulus expectancy effects on pain by comparing the BOLD signal associated with positive expectations (i.e., LM condition) and negative expectations (i.e., HM condition) versus that during the MM condition. We first analyzed the contrast " $(\mathrm{LM}+\mathrm{HM})>2 \mathrm{MM}$ " to search for brain regions modulated by expectations regardless of the valence, but this analysis did not produce any significant clusters. We then examined brain regions respectively modulated by positive (contrast "LM > MM") and negative (contrast "HM > MM") expectations. We found that positive expectations entailed a significant increase in activation in the right aIC (peak MNI coordinates $x / y / z=34 / 26 / 10, t_{(1,20)}=5.91, p=0.001$; SVC, 


\section{Contrast "LM > MM"}

\section{A Activation}
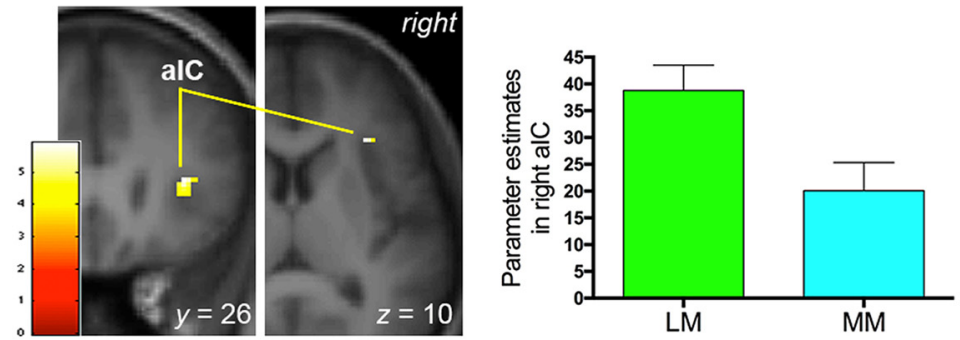

B PPI (increased connectivity)
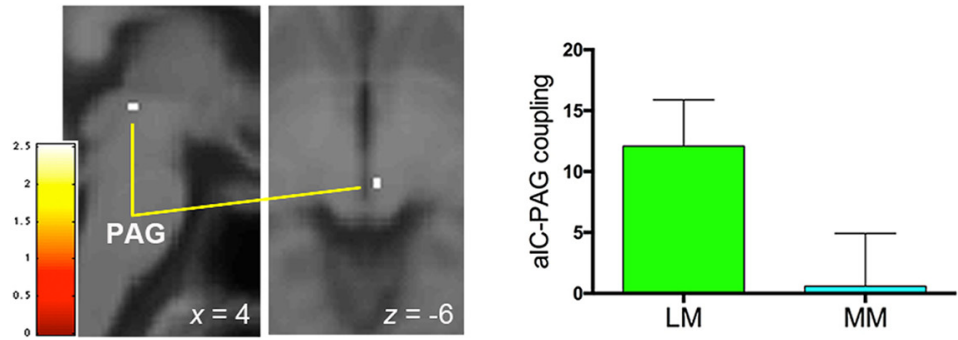

C PPI: correlation with pain ratings
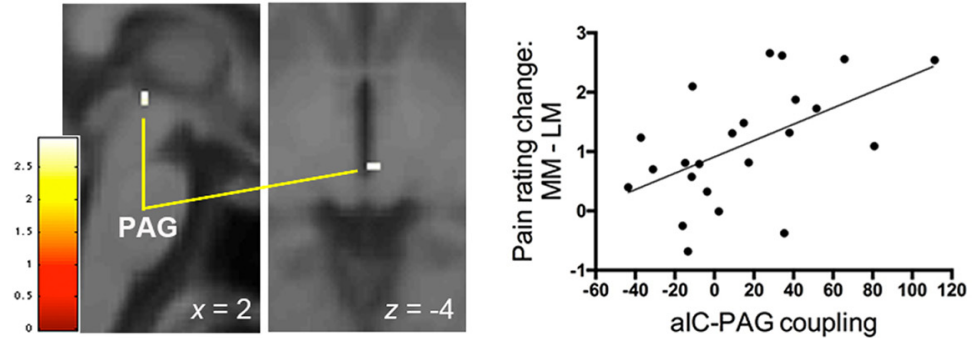

D PPI: correlation with prediction errors
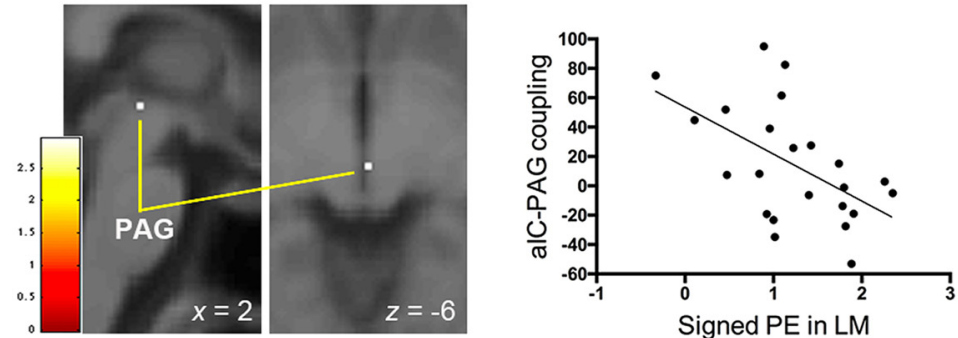

E PPI: correlation with certainty ratings
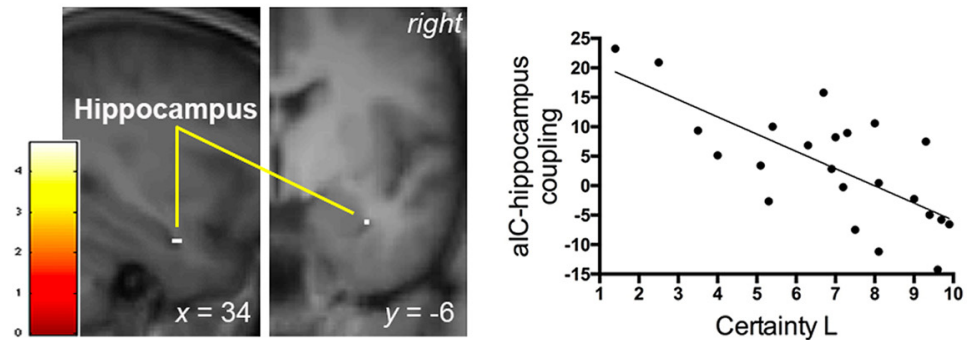

Figure 3. Neural mechanisms associated with positive expectancy effects on pain. This figure shows the results of brain activation and PPI analyses in the right alC associated with positive expectancy effects on pain. $\boldsymbol{A}$, The right alC exhibited increased activation as positive expectations reduced subjective pain perception. $\boldsymbol{B}$, Positive expectations were also accompanied by an increase in functional connectivity between the right alC and PAG. C, Functional connectivity between the right alC and PAG predicted the magnitude of pain rating changes caused by positive expectations. $\boldsymbol{D}$, The signed prediction error (PE) of pain engendered in the LM condition was inversely associated with the aIC-PAG connectivity associated with positive expectations
FWE-corrected; Fig. 3A) and were not associated with significant activation or deactivation in rACC, PAG or VS. In a whole-brain analysis, only a cluster in the right aIC survived a whole-brain correction for multiple comparisons (cluster size $=177$ voxels, peak MNI coordinates $x / y / z=36 / 24 / 12, t_{(1,20)}=6.36, p=0.047$; FWE-corrected). Right aIC activation remained significant when we directly compared the BOLD signals between positive and negative expectations (i.e., contrast "LM > HM": peak MNI coordinates $x / y /$ $z=32 / 30 / 8, t_{(1,20)}=5.17, p=0.004 ;$ SVC, FWE-corrected). By contrast, negative expectations produced a significant increase in the right rACC (peak MNI coordinates $x / y / z=0 / 34 / 22, t_{(1,20)}=3.92, p=0.039$; SVC, FWE-corrected; Fig. $4 A$ ) and were not associated with significantly enhanced or reduced BOLD signals in aIC, PAG, or VS. The conjunction analysis of "LM > MM" and "HM > MM" contrasts did not produce any significant voxels. Interestingly, consistent with the behavioral data (Fig. $1 D$ ), the BOLD signal in the right aIC associated with positive expectations was significantly correlated with that in the right rACC related to negative expectations $(r=0.483 ; p=0.019$; Fig. 5$)$. To determine whether the neural substrate coding pain intensity per se contributed to positive expectation- and negative expectation-related pain modulation, we also used the suprathreshold cluster from the whole-brain analysis of contrast "NL $<\mathrm{NM}<\mathrm{NH}$ " (Fig. $2 A$ ) as a mask to perform SVCs for contrasts "LM $>$ MM" and "HM > MM," but neither of these analyses produced significant clusters.

\section{Functional connectivity with pain-processing circuits}

The above findings suggest that positive and negative expectations recruited different neural substrates to modulate pain. If these regions indeed underlay stimulus expectancy effects on pain, we would expect functional interactions between these regions and key pain-processing brain structures. To test this hypothesis, we then

$\leftarrow$

$\boldsymbol{E}$, Functional connectivity between the right alC and right hippocampus was inversely correlated with subjective certainty ratings elicited by a low-pain cue. $\boldsymbol{A}-\boldsymbol{E}$, Activation clusters survived small-volume corrections ( $p<0.05$, FWE corrected) and were overlapped on an average structural image. The bar on the left shows the range of $t$ scores for SPM 8. Bar graphs and scatter plots depict parameter estimates extracted from the suprathreshold cluster. Error bars in $\boldsymbol{A}$ and $\boldsymbol{B}$ represent SEM. Scatter plots depict the relationship between behavioral data and the strength of functional connectivity. 


\section{Contrast "HM > MM"}

\section{A Activation}
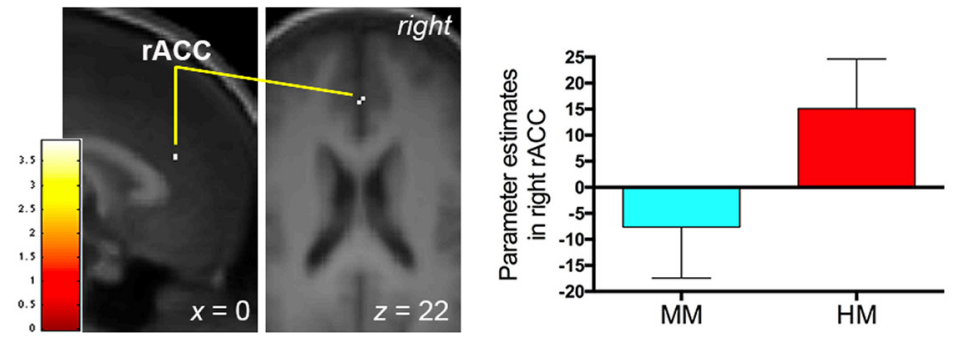

B PPI (reduced connectivity)
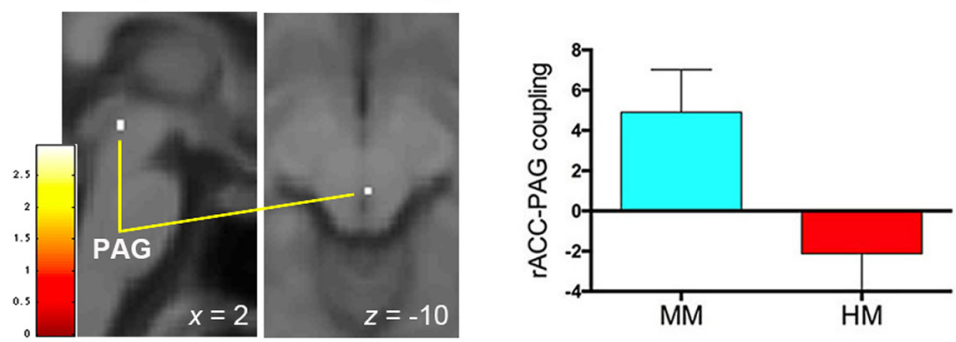

C PPI: correlation with pain ratings
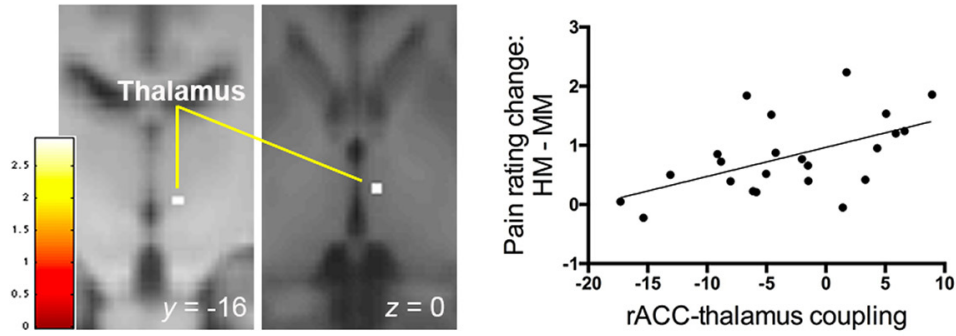

D PPI: correlation with prediction errors
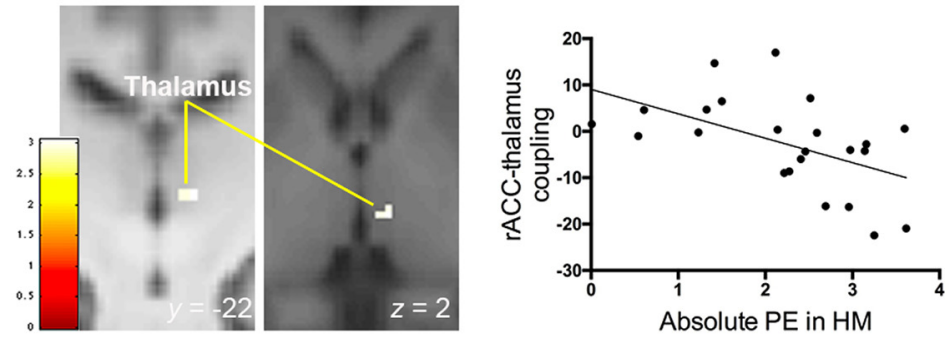

E PPI: correlation with certainty ratings
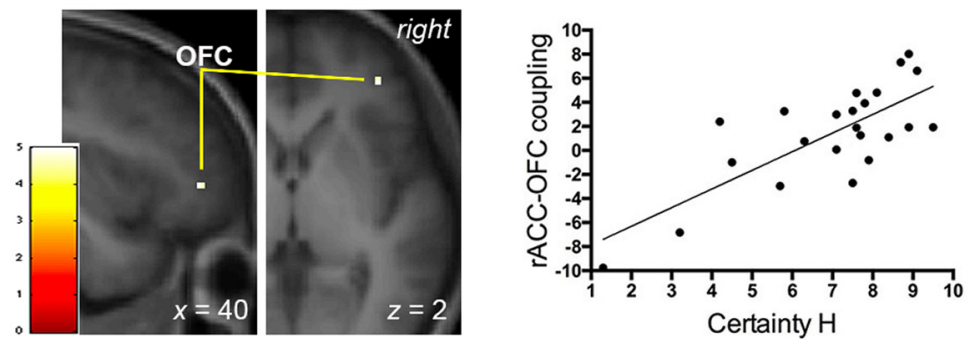

Figure 4. Neural mechanisms associated with negative expectancy effects on pain. This figure shows the results of brain activation and PPI analyses in the right rACC associated with negative expectancy effects on pain. $A$, Pain modulation by negative expectations entailed increased activation in the right rACC. $\boldsymbol{B}$, Negative expectations were also accompanied by reduced functional connectivity between the $\mathrm{rACC}$ and PAG. C, Functional connectivity between the right rACC and right thalamus predicted the magnitude of pain rating changes provoked by negative expectations. $\boldsymbol{D}$, The absolute prediction error (PE) of pain engendered in the HM condition was inversely correlated with the $\mathrm{rACC}$-thalamus functional connectivity associated with negative expectations. $\boldsymbol{E}$, Functional connectivity between the right rACC and right lateral OFC covaried with subjective certainty ratings elicited by a performed PPI analyses using the right aIC and right rACC as seed.

We found that the right aIC and right rACC exhibited opposite functional connectivity with the PAG, a descending pain modulatory control center, as expectations modulated pain perception. Positive expectations (i.e., contrast "LM > MM") entailed a significant increase in the coupling between the right aIC and right PAG (peak MNI coordinates $x / y / z=4 /-28 /-6$, $t_{(1,22)}=2.53, p=0.049$; SVC, FWE-corrected; Fig. $3 B$ ). A group-level regression analysis using individual changes in pain ratings (i.e., MM-LM) as a covariate further revealed that the coupling between right aIC and right PAG (peak MNI coordinates $x / y / z=2 /-26 /-4, t_{(1,21)}=2.95$, $p=0.022$; SVC, FWE-corrected) increased as a linear function of pain rating changes under positive expectations (Fig. $3 C)$. By contrast, negative expectations (i.e., contrast "HM > MM") were accompanied by a significant decrease in the coupling between the right rACC and right PAG (peak MNI coordinates $x / y / z=$ $2 /-30 /-10, t_{(1,22)}=2.96, p=0.020$; SVC, FWE-corrected; Fig. 4B). Here the activation peak in the PAG was located ventrally adjacent to that observed during positive expectations (i.e., 4/-28/-6; Fig. $3 B)$. By correlating individual changes in pain ratings during negative expectations (i.e., HM-MM), we found that pain rating changes covaried significantly with the connectivity from the rACC to the right thalamus (peak MNI coordinates $x / y / z=$ $6 /-16 / 0, t_{(1,21)}=2.91, p=0.037$; SVC, FWE-corrected; Fig. $4 C$ ), the relay center in the ascending pain pathway. The peak coordinates of the right thalamus identified here corresponded closely to those associated with intensity coding described above (i.e., 8/-18/-2). Across subjects, the strength of negative expectationrelated connectivities with rACC (i.e., rACC-PAG and rACC-thalamus connectivities in Fig. $4 B, C$ ) did not significantly covary with that of positive expectationassociated connectivity with aIC (i.e., aIC-PAG connectivity in Fig. $3 B, C$; all $p>0.082)$.

high-pain cue. $\boldsymbol{A}-\boldsymbol{E}$, Activation clusters survived small-volume corrections ( $p<0.05$, FWE corrected) and were overlapped on an average structural image. The bar on the left shows the range of $t$ scores for SPM 8. Bar graphs and scatter plots depict parameter estimates extracted from the suprathreshold cluster. Error bars in $\boldsymbol{A}$ and $\boldsymbol{B}$ represent SEM. Scatter plots depict the relationship between behavioral data and the strength of functional connectivity. 


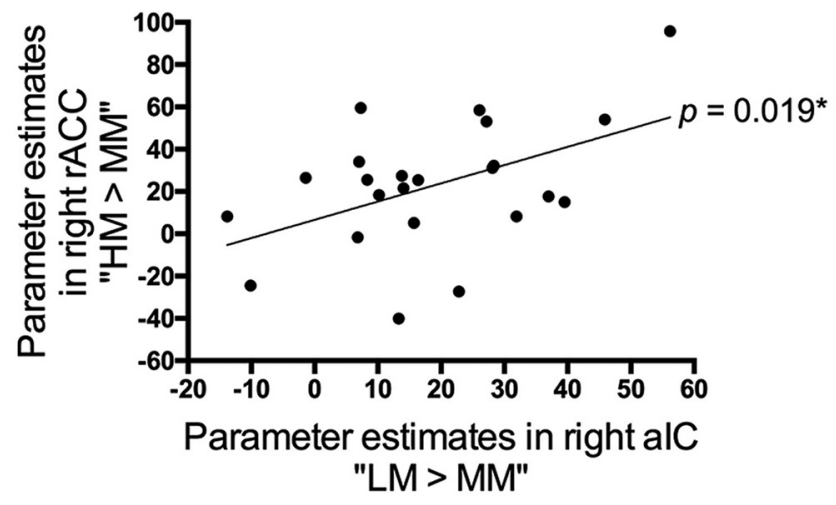

Figure 5. Linear relationship between positive expectation- and negative expectationassociated brain activations. The BOLD signal within the significant cluster in the right alC (contrast "LM $>M M^{\prime \prime}$ ) increased monotonically with that in the right rACC (contrast "HM > $\left.M M^{\prime \prime}\right)$ across subjects $(p=0.019)$. Data in this scatter plot were parameter estimates extracted from the suprathreshold cluster of the contrast "LM $>M M^{\prime \prime}$ (Fig. 3A) and "HM $>M M$ " (Fig. 4A). ${ }^{*} p<0.05$.

\section{Prediction error mechanisms underlying stimulus expectancy} effects on pain

Previous research has reported that stimulus-expectancyassociated brain regions may engage a variety of cognitive processes to modulate pain (Atlas et al., 2010). Concerning the prediction error account, both the signed mismatch (Rescorla and Wagner, 1972; Sutton and Barto, 1998) and absolute (unsigned) mismatch (Pearce and Hall, 1980; Courville et al., 2006) between received and expected outcome have been proposed to affect expectations and brain regions coding signed and absolute prediction errors have been reported in previous neuroimaging studies in humans (Ploghaus et al., 2000; O’Doherty et al., 2003; Geuter et al., 2017; Fazeli and Büchel, 2018). Theoretically, compared with the outcome that does not violate expectations (e.g., $\mathrm{MM}$ in our case), brain areas coding signed prediction errors of pain would show an increased response when receiving a higherthan-expected outcome (e.g., LM) and a decreased response when receiving a lower-than-expected outcome (e.g., HM, LL, and $\mathrm{HH}$ ). Brain regions that are only sensitive to positive prediction errors (Fig. 6A) (McClure et al., 2003; Kim et al., 2006; Yacubian et al., 2006; Seymour et al., 2007) would also show an increased response when receiving a higher-than-expected outcome, but would not entail a decreased response when receiving a lower-than-expected outcome. For those representing absolute prediction errors, the activation would be higher when the expected pain intensity violates received pain (e.g., LM, HM, LL, and $\mathrm{HH}$ ) relative to the condition without expectancy violation (e.g., MM; Fig. 6B).

To investigate whether brain regions underlying stimulus expectancy effects on pain implemented prediction error processing, we then inspected the response profiles of the right aIC and right rACC across the five cued conditions in our paradigm. We found that the BOLD signal within the right aIC (Fig. $3 A$ ) during the LM condition was significantly higher than the LL ( $p=$ $0.037)$, HM $(p=0.001)$, and HH $(p=0.010)$ conditions, with comparable responses between the MM and HM conditions ( $p=$ 0.581 ; Fig. $6 A$ ). These findings suggest the possibility that a positive prediction error signal of pain was engendered in the right aIC. As for the right rACC, its BOLD signal (Fig. 4A) could be described as a combination of absolute prediction errors of pain, which explained the significant difference in BOLD signals between LM and MM conditions $(p=0.047)$, and nociceptive processing, which explained the significantly higher response in the $\mathrm{HH}$ condition versus the LL $(p<0.0001)$, LM $(p=0.041)$, and MM ( $p=0.003)$ conditions, as well as the comparable responses between HM and $\mathrm{HH}$ conditions ( $p=0.152$; Fig. $6 B$ ).

If these findings indeed reflect a role of aversive prediction errors in stimulus expectancy effects on pain, then it would be expected that the functional interaction between stimulus expectancy- and pain processing-related regions (i.e., aIC-PAG coupling for positive expectations and rACC-thalamus coupling for negative expectations) would show modulation by prediction errors. Importantly, we found that, across participants, the prediction error associated with positive expectations was negatively correlated with the strength of aIC-PAG coupling under positive expectations (i.e., contrast "LM > MM; signed prediction errors: $r=-0.515, p=0.012$; absolute prediction errors: $r=-0.492$, $p=0.017)$. Consistent with this finding, the SVC analysis in a simple linear regression model in SPM also showed that prediction errors of pain inversely predicted the connectivity from the right aIC to the right PAG (signed prediction errors: peak MNI coordinates $x / y / z=2 /-26 /-6, t_{(1,22)}=2.95, p=0.022$, Fig. $3 D$; absolute prediction errors: peak MNI coordinates $x / y / z=2 /-26 /$ $-6, t_{(1,22)}=2.77, p=0.031$; SVC, FWE-corrected). Regarding negative expectations, we tested whether the absolute prediction errors of pain in the HM condition and the reported pain rating for the NH condition together influenced rACC-thalamus coupling, given that the response pattern in the right rACC appeared to be the combination of absolute prediction errors and nociceptive processing (Fig. 6B). The multiple linear regression analysis showed that both the absolute prediction errors of pain $(\beta=$ $-0.465, t=-2.565, p=0.018)$ and the reported pain rating $(\beta=-0.467, t=-2.576, p=0.018)$ significantly predicted the strength of rACC-thalamus coupling $\left(F_{(2,20)}=5.668, r^{2}=0.362\right.$, $p=0.011)$. In a multiple linear regression model in SPM, the prediction error of pain significantly and inversely predicted the magnitude of connectivity from the rACC to the right thalamus (absolute prediction errors: peak MNI coordinates $x / y / z=$ $8 /-22 / 2, t_{(1,22)}=3.06, p=0.030$; SVC, FWE-corrected; Fig. 4D) after regressing out the effect of reported pain rating. Analyses using reported pain ratings for the $\mathrm{HH}$ condition produced similar results.

\section{Effect of between-subject differences in subjective certainty about expectations}

Finally, because participants' self-reported certainty (Fig. 1G) and anxiety (Fig. $1 J$ ) about predicted pain intensity influenced the modulation of stimulus expectancies on pain, we explored the neural streams underlying the impact of these psychological factors on pain expectations. If the identified brain regions (i.e., right aIC, Fig. $3 A$, and rACC, Fig. $4 A$ ) did contribute to the modulatory effect of expectations, we predicted that these regions would collaborate with the hippocampus, a brain region implicated in pain-related anxiety (Ploghaus et al., 2001; Kong et al., 2008; Gondo et al., 2012; Freeman et al., 2015), to reflect the influence of pain anticipation-related anxiety on expectationinduced pain modulation, especially during positive expectations (Fig. $1 J$ ). We also anticipated that brain regions subserving stimulus expectancy effects on pain would interact with the lateral OFC, a brain structure associated with the outcome value and certainty (Rogers et al., 1999; Critchley et al., 2001; PadoaSchioppa and Assad, 2006; Rudebeck and Murray, 2014; Winston et al., 2014), to represent the impact of anticipation-related cer- 
A
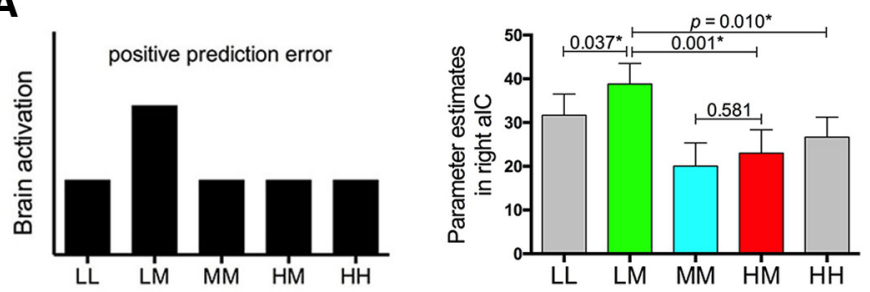

B
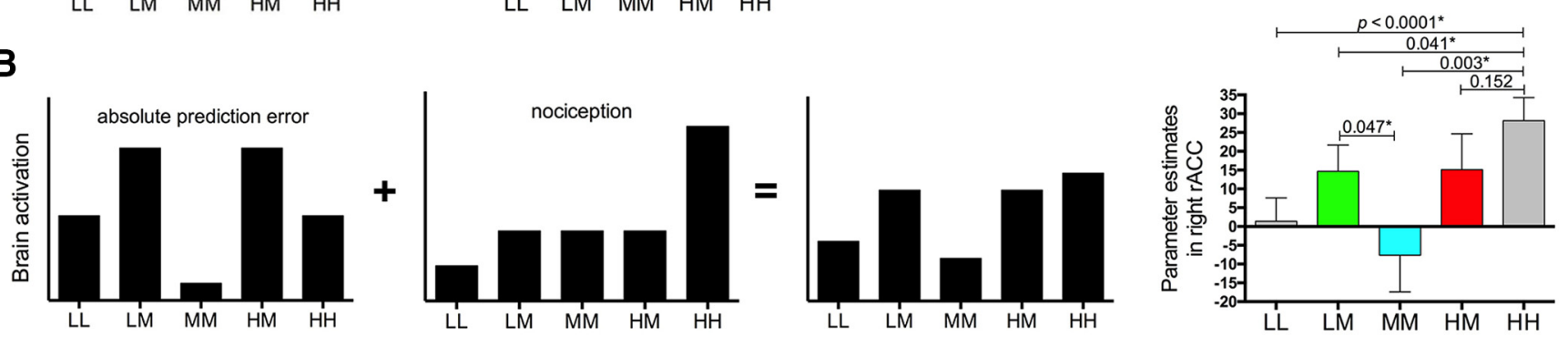

Figure 6. Response patterns in key regions underlying stimulus expectancy effects on pain. Black bars depict theoretical pattern of B0LD signals across different trial types (equal weighting of both components in $\boldsymbol{B})$. $\boldsymbol{A}$, The right alC possibly engendered a positive prediction error of pain because its response during the LM condition was significantly stronger than all the rest four conditions and there was no significant difference between the HM and MM conditions. $\boldsymbol{B}$, The response profile of the right rACC could be accounted for by an absolute pain prediction error component (highest responses in LM and $\mathrm{HM}$, lowest in $\mathrm{MM}$, with $\mathrm{LL}$ and $\mathrm{HH}$ in the middle) plus a nociception component (highest for high-pain stimulation, followed in descending order by middle- and low-pain stimulation). For the alC and rACC, the BOLD signals were extracted from the suprathreshold cluster of the contrast "LM $>M M$ " (Fig. $3 A$ ) and "HM $>M M$ " (Fig. $4 A$ ), respectively. ${ }^{*} p<0.05$.

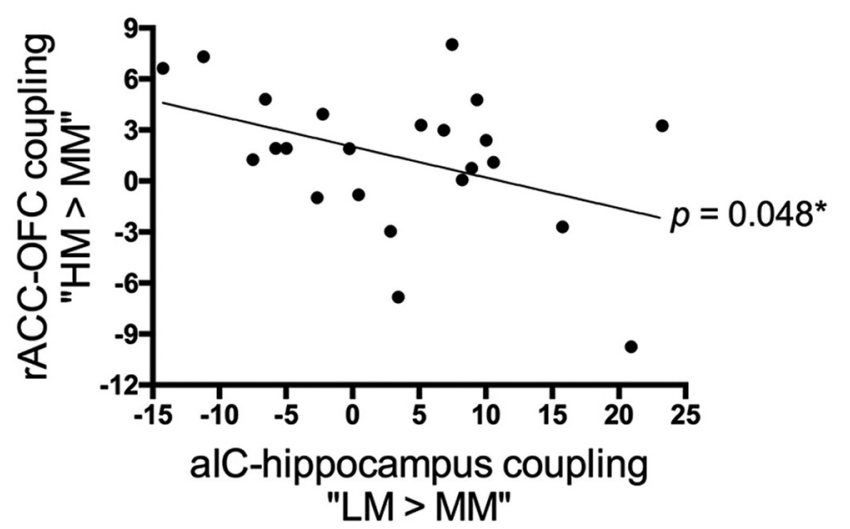

Figure 7. Linear relationship in certainty-related functional connectivities between positive and negative expectations. Across subjects, positive expectation-associated functional connectivity between the right alC and right hippocampus was inversely correlated with negative expectation-associated functional connectivity between the right rACC and right lateral OFC $(p=0.048)$. Data in this scatter plot were parameter estimates extracted from the suprathreshold cluster in Figures $3 E$ and $4 E .{ }^{*} p<0.05$.

tainty on stimulus expectancy effects on pain. Moreover, we tested whether the revealed mechanisms were interrelated between positive and negative expectations.

Consistent with our hypothesis, the strength of the functional connectivity from the right aIC to the right hippocampus (contrast "LM > MM": peak MNI coordinates $x / y / z=34 /-6 /-24$, $t_{(1,21)}=4.71, p=0.049$; SVC, FWE-corrected; Fig. $\left.3 E\right)$ was found to be inversely correlated with participants' certainty ratings under positive expectations. As for negative expectations, the right rACC coupled with the right lateral OFC (contrast "HM $>M M$ ": peak MNI coordinates $x / y / z=40 / 42 / 2, t_{(1,21)}=5.00, p=0.013$; SVC, FWE-corrected; Fig. $4 E$ ) to reflect the modulation of certainty across subjects. Importantly, the strength of the aIC-hippocampus connectivity under positive expectations showed a significant negative correlation with that of $\mathrm{rACC}-\mathrm{OFC}$ connectivity under negative expectations $(r=-0.417 ; p=0.048$; Fig. 7$)$.

\section{Discussion}

Here, we demonstrated that positive and negative expectations entailed separate activations in aversive prediction-error-related regions. These pain modulation effects covaried with participants' certainty about expectations, which involved the functional connectivities between aversive prediction-error-related regions and brain regions implicated in outcome certainty and anxiety. Intriguingly, these certainty-associated functional connectivities, pain rating changes, and brain activation associated with stimulus expectancy effects on pain all showed a linear relationship between positive and negative expectations. These results suggest that different but interrelated aversive prediction error signals underlie positive and negative expectancy effects on pain and individual certainty about expectations dependently regulate both types of pain modulation.

\section{Aversive prediction error signals in stimulus expectancy effects on pain}

Despite a considerable amount of research on reward prediction errors (Schultz et al., 1997; Schultz, 2016), relatively little is known about the functional significance of aversive prediction error signals. The finding that the VS did not reflect prediction errors of pain during both positive and negative expectations echoes a recent study showing that prediction error processing in the VS would become dysfunctional when participants' perception to pain was strongly modulated by expectations (Schenk et al., 2017). Intriguingly, we revealed that brain regions implicated in aversive prediction errors, specifically the aIC and rACC, participated in cue-based stimulus expectancy effects on pain and differentiated positive from negative expectations. Consistent with previous research (Seymour et al., 2004, 2005), we found that the aIC involved in positive expectation-induced pain modulation represented the positive prediction error of pain. By contrast, the rACC that underlay the effect of negative expectations reflected the absolute prediction error of pain, which is consistent with previous observation that receiving a lower-than-expected pain entails rACC activation (Leknes et al., 2011; Navratilova et al., 2015). These findings thus extend the role of prediction error in pain processing, indicating that pain-related prediction error 
signals not only underlie pain perception (Geuter et al., 2017) and placebo hypoalgesia (Schenk et al., 2017), but also subserve stimulus expectancy effects on pain. Because the trial-by-trial prediction error was not collected in our paradigm, future studies investigating trialwise neural fluctuations in prediction error coding would help to clarify whether the sustained pain modulation effect by stimulus expectancy observed in the current study stems from impaired updating of future expectations for pain.

\section{Pain modulation mechanisms associated with positive and negative expectations}

The PPI finding that the aIC and $\mathrm{rACC}$ coupled with the PAG and thalamus not only indicates that stimulus expectancy effects on pain arise from the interaction between brain regions representing neuronal error signals for pain and pain-processing circuits, but also fits well with the perspective that the modulation of expectations on pain is implemented in a hierarchical system encompassing ascending and descending pain pathways (Büchel et al., 2014). Interestingly, positive and negative expectations exhibited opposite coupling with PAG, with an increased aIC-PAG coupling under positive expectations but a decreased rACC-PAG coupling under negative expectations. The coupling from the right aIC and right $\mathrm{rACC}$ to the right PAG is in good accordance with the predominantly ipsilateral descending projections from both aIC and rACC to PAG (Mantyh, 1982; Floyd et al., 2000). Following this finding, we observed that the aIC-PAG coupling further reflected the degree to which positive expectations biased pain perception, whereas the rACC coactivated with the right thalamus to reflect the impact of negative expectations. Of note, both aIC-PAG and rACC-thalamus connectivities covaried with the prediction error of pain. Given that the PAG influences descending pain modulation (Tracey and Mantyh, 2007) and has recently been demonstrated to represent prediction errors of pain (Roy et al., 2014), these findings reinforce the notion that aversive prediction error signals underlie stimulus expectancy effects on pain. Anatomically, fibers from aIC and rACC project to the PAG to subserve top-down cognitive modulation of pain (Tracey and Mantyh, 2007) and rACC receives bottom-up thalamic afferents to process the affective aspects of pain (Price, 2000). Therefore, our data provide direct evidence that positive expectations reduce pain by enhancing the interaction between the neural system encoding aversive prediction errors and descending pain inhibitory system. By contrast, negative expectations appear to nonlinearly increase pain by reducing this interaction. Because negative expectations enhanced pain perception, the revealed augmented rACC-thalamus coupling may reflect the increased pain aversiveness during this process.

\section{Modulatory effect of subjective certainty about expectations}

Consistent with the modulatory effect of participants' certainty about expectations on pain perception, further analyses revealed that pain prediction-error-related regions collaborated with the neural substrate related to outcome certainty and anxiety to modulate stimulus expectancy effects on pain. As a low-pain cue emerged, participants reporting a higher level of certainty about their predictions, which was accompanied by a lower anxiety level, exhibited a weaker aIC-hippocampus coupling and a greater amount of pain reduction during painful stimulation. aIC has anatomical connections with hippocampus (Augustine, 1996; Cerliani et al., 2012) and both aIC and hippocampus have been implicated in the modulatory effect of certainty on pain (Brown et al., 2008). Hippocampus has long been thought to regulate anxiety (Bannerman et al., 2014), including pain-related anxiety (Ploghaus et al., 2001; Gondo et al., 2012; Freeman et al., 2015). Combined with a selective pain modulatory effect of anxiety during positive expectations in our behavioral data, we posit that the increased certainty (and thus lowered anxiety) about positive expectations downregulates the functional interaction between the aIC and hippocampus, thereby generating an anxiolytic effect and thus dampening the amplification of pain signaling in hippocampus (Gray and McNaughton, 2000; Hasler et al., 2007).

With regard to negative expectations, we observed that, as a high-pain cue emerged, participants reporting a higher level of certainty about their predictions showed a stronger $\mathrm{rACC}-\mathrm{OFC}$ coupling during painful stimulation, which was accompanied by a greater extent of pain increase as well as a larger difference between expected and reported pain intensities. The rACC has been assumed to underlie the effect of certainty on pain (Brown et al., 2008) and both the rACC and lateral OFC have been demonstrated to reflect anticipation-related outcome certainty (Rogers et al., 1999; Critchley et al., 2001). The lateral OFC has also been linked to the processing of the threat value of an incoming stimulation (Wiech et al., 2010) and effects of negative expectations on pain (Freeman et al., 2015). As a convergence center that receives afferents from both the somatosensory and limbic association areas including the rACC (Morecraft et al., 1992), neurons within the lateral OFC have been shown to assign subjective value to external stimuli including pain (Padoa-Schioppa and Assad, 2006; Rudebeck and Murray, 2014; Winston et al., 2014). Given that a high-pain cue was followed by either a high- or a medium-pain stimulation in our paradigm and that a painful stimulus would be perceived as pleasant if its intensity is relatively low in the context (Leknes et al., 2013), we speculate that subjective certainty under negative expectations regulates the conversion of a painful stimulus into a positive hedonic value (i.e., the pleasant sensation arising from the receipt of a relatively low intensity of pain stimulation). The higher the subjective certainty about negative expectations, the lower the reported pain intensity compared with the expected pain intensity and thus a larger positive hedonic value assigned to this stimulation.

\section{Relationship between positive and negative expectations}

Although it remains unclear whether stimulus expectancy- and treatment expectancy-associated pain modulation engages convergent mechanisms (Atlas and Wager, 2014), we observed a significant association in both pain rating changes and cerebral activation between positive and negative expectations, which is different from a recent study investigating effects of treatmentassociated positive versus negative expectations on pain (Freeman et al., 2015). Moreover, we discovered that subjective certainty about expectations comparably modulated the impact of positive and negative expectations on pain perception. The finding that these modulations were subserved by two correlated functional connectivities (i.e., aIC-hippocampus and rACCOFC connectivities) suggests that modulation of subjective certainty on positive and negative expectations is interrelated at the neural level. Together with the common interaction with the PAG as discussed above, we thus argue that positive and negative expectations are two separate but interrelated and dependently regulated cognitive constructs, which converge in the descending pain modulatory system to shape human pain experiences. Based on the notion that stimulus expectancies are instant predictions about outcomes in the environment (Atlas and Wager, 2014), we propose that individual certainty may act as a metacognitive process implemented in the correlated aIC-hippocampus and rAC- 
C-OFC connectivities to enable quick switching between positive and negative expectations.

In conclusion, we provide evidence that aversive prediction error signals underlie stimulus expectancy effects on pain and positive expectation- and negative expectation-related modulation mechanisms are different but dependently regulated by subjective certainty. These mechanisms help to explain why we humans can adapt quickly and appropriately to noxious stimuli the intensity of which deviates from our expectations. Given that electrical stimulation coactivates non-nociceptive afferents, future studies using different pain modalities as well as nonpainful aversive stimuli will help to clarify the pain specificity of these identified mechanisms.

\section{References}

Apkarian AV, Bushnell MC, Treede RD, Zubieta JK (2005) Human brain mechanisms of pain perception and regulation in health and disease. Eur J Pain 9:463-484. CrossRef Medline

Atlas LY, Wager TD (2014) A meta-analysis of brain mechanisms of placebo analgesia: consistent findings and unanswered questions. Handb Exp Pharmacol 225:37-69. CrossRef Medline

Atlas LY, Bolger N, Lindquist MA, Wager TD (2010) Brain mediators of predictive cue effects on perceived pain. J Neurosci 30:12964-12977. CrossRef Medline

Augustine JR (1996) Circuitry and functional aspects of the insular lobe in primates including humans. Brain Res Rev 22:229-244. CrossRef Medline

Bannerman DM, Sprengel R, Sanderson DJ, McHugh SB, Rawlins JN, Monyer H, Seeburg PH (2014) Hippocampal synaptic plasticity, spatial memory and anxiety. Nat Rev Neurosci 15:181-192. CrossRef Medline

Brown CA, Seymour B, El-Deredy W, Jones AK (2008) Confidence in beliefs about pain predicts expectancy effects on pain perception and anticipatory processing in right anterior insula. Pain 139:324-332. CrossRef Medline

Büchel C, Geuter S, Sprenger C, Eippert F (2014) Placebo analgesia: a predictive coding perspective. Neuron 81:1223-1239. CrossRef Medline

Cerliani L, Thomas RM, Jbabdi S, Siero JC, Nanetti L, Crippa A, Gazzola V, D’Arceuil H, Keysers C (2012) Probabilistic tractography recovers a rostrocaudal trajectory of connectivity variability in the human insular cortex. Hum Brain Mapp 33:2005-2034. CrossRef Medline

Courville AC, Daw ND, Touretzky DS (2006) Bayesian theories of conditioning in a changing world. Trends Cogn Sci 10:294-300. CrossRef Medline

Craig AD (2003) Pain mechanisms: labeled lines versus convergence in central processing. Annu Rev Neurosci 26:1-30. CrossRef Medline

Critchley HD, Mathias CJ, Dolan RJ (2001) Neural activity in the human brain relating to uncertainty and arousal during anticipation. Neuron 29:537-545. CrossRef Medline

Delgado MR, Li J, Schiller D, Phelps EA (2008) The role of the striatum in aversive learning and aversive prediction errors. Philos Trans R Soc Lond B Biol Sci 363:3787-3800. CrossRef Medline

Duerden EG, Albanese MC (2013) Localization of pain-related brain activation: a meta-analysis of neuroimaging data. Hum Brain Mapp 34:109149. CrossRef Medline

Eklund A, Nichols TE, Knutsson H (2016) Cluster failure: why fMRI inferences for spatial extent have inflated false-positive rates. Proc Natl Acad Sci U S A 113:7900-7905.

Ezra M, Faull OK, Jbabdi S, Pattinson KT (2015) Connectivity-based segmentation of the periaqueductal gray matter in human with brainstem optimized diffusion MRI. Hum Brain Mapp 36:3459-3471. CrossRef Medline

Fairhurst M, Wiech K, Dunckley P, Tracey I (2007) Anticipatory brainstem activity predicts neural processing of pain in humans. Pain 128:101-110. CrossRef Medline

Faull OK, Pattinson KT (2017) The cortical connectivity of the periaqueductal gray and the conditioned response to the threat of breathlessness. Elife 6:21749. CrossRef Medline

Fazeli S, Büchel C (2018) Pain related expectation and prediction error signals in the anterior insula are not related to aversiveness. J Neurosci 38:6461-6474. CrossRef Medline
Floyd NS, Price JL, Ferry AT, Keay KA, Bandler R (2000) Orbitomedial prefrontal cortical projections to distinct longitudinal columns of the periaqueductal gray in the rat. J Comp Neurol 422:556-578. CrossRef Medline

Freeman S, Yu R, Egorova N, Chen X, Kirsch I, Claggett B, Kaptchuk TJ, Gollub RL, Kong J (2015) Distinct neural representations of placebo and nocebo effects. Neuroimage 112:197-207. CrossRef Medline

Friston KJ, Holmes AP, Worsley KJ, Poline JP, Frith CD, Frackowiak RS (1995) Statistical parametric maps in functional imaging: a general linear approach. Hum Brain Mapp 2:189-210.

Friston KJ, Buechel C, Fink GR, Morris J, Rolls E, Dolan RJ (1997) Psychophysiological and modulatory interactions in neuroimaging. Neuroimage 6:218-229. CrossRef Medline

Garrison J, Erdeniz B, Done J (2013) Prediction error in reinforcement learning: a meta-analysis of neuroimaging studies. Neurosci Biobehav Rev 37:1297-1310. CrossRef Medline

Geuter S, Boll S, Eippert F, Büchel C (2017) Functional dissociation of stimulus intensity encoding and predictive coding of pain in the insula. Elife 6:e24770. CrossRef Medline

Gondo M, Moriguchi Y, Kodama N, Sato N, Sudo N, Kubo C, Komaki G (2012) Daily physical complaints and hippocampal function: an fMRI study of pain modulation by anxiety. Neuroimage 63:1011-1019. CrossRef Medline

Gray JA, McNaughton N (2000) The neuropsychology of anxiety, Ed 2. Oxford: OUP.

Greenspan JD, Craft RM, LeResche L, Arendt-Nielsen L, Berkley KJ, Fillingim RB, Gold MS, Holdcroft A, Lautenbacher S, Mayer EA, Mogil JS, Murphy AZ, Traub RJ; Consensus Working Group of the Sex, Gender, and Pain SIG of the IASP (2007) Studying sex and gender differences in pain and analgesia: a consensus report. Pain 132:S26-S45. CrossRef Medline

Hasler G, Fromm S, Alvarez RP, Luckenbaugh DA, Drevets WC, Grillon C (2007) Cerebral blood flow in immediate and sustained anxiety. J Neurosci 27:6313-6319. CrossRef Medline

Herry C, Johansen JP (2014) Encoding of fear learning and memory in distributed neuronal circuits. Nat Neurosci 17:1644-1654. CrossRef Medline

Holmes AP, Friston KJ (1998) Generalisability, random effects and population inference. Neuroimage 7:S754. CrossRef

Hsieh JC, Stone-Elander S, Ingvar M (1999) Anticipatory coping of pain expressed in the human anterior cingulate cortex: a positron emission tomography study. Neurosci Lett 262:61-64. CrossRef Medline

Hutton C, Bork A, Josephs O, Deichmann R, Ashburner J, Turner R (2002) Image distortion correction in $\mathrm{fMRI}$ : a quantitative evaluation. Neuroimage 16:217-240. CrossRef Medline

Keltner JR, Furst A, Fan C, Redfern R, Inglis B, Fields HL (2006) Isolating the modulatory effect of expectation on pain transmission: a functional magnetic resonance imaging study. J Neurosci 26:4437-4443. CrossRef Medline

Kim H, Shimojo S, O'Doherty JP (2006) Is avoiding an aversive outcome rewarding? neural substrates of avoidance learning in the human brain. PLoS Biol 4:e233. CrossRef Medline

Kong J, Gollub RL, Polich G, Kirsch I, Laviolette P, Vangel M, Rosen B, Kaptchuk TJ (2008) A functional magnetic resonance imaging study on the neural mechanisms of hyperalgesic nocebo effect. J Neurosci 28 : 13354-13362. CrossRef Medline

Kong J, Jensen K, Loiotile R, Cheetham A, Wey HY, Tan Y, Rosen B, Smoller JW, Kaptchuk TJ, Gollub RL (2013) Functional connectivity of the frontoparietal network predicts cognitive modulation of pain. Pain 154:459 467. CrossRef Medline

Koyama T, McHaffie JG, Laurienti PJ, Coghill RC (2005) The subjective experience of pain: where expectations become reality. Proc Natl Acad Sci U S A 102:12950-12955. CrossRef Medline

Leknes S, Lee M, Berna C, Andersson J, Tracey I (2011) Relief as a reward: hedonic and neural responses to safety from pain. PLoS One 6:e17870. CrossRef Medline

Leknes S, Berna C, Lee MC, Snyder GD, Biele G, Tracey I (2013) The importance of context: when relative relief renders pain pleasant. Pain 154: 402-410. CrossRef Medline

Mantyh PW (1982) Forebrain projections to the periaqueductral gray in the monkey, with observations in the cat and rat. J Comp Neurol 206:146158. CrossRef Medline

McClure SM, Berns GS, Montague PR (2003) Temporal prediction errors in 
a passive learning task activate human striatum. Neuron 38:339-346. CrossRef Medline

McLaren DG, Ries ML, Xu G, Johnson SC (2012) A generalized form of context-dependent psychophysiological interactions (gPPI): a comparison to standard approaches. Neuroimage 61:1277-1286. CrossRef Medline

McNally GP, Johansen JP, Blair HT (2011) Placing prediction into the fear circuit. Trends Neurosci 34:283-292. CrossRef Medline

Morecraft RJ, Geula C, Mesulam MM (1992) Cytoarchitecture and neural afferents of orbitofrontal cortex in the brain of the monkey. J Comp Neurol 323:341-358. CrossRef Medline

Morgan MM, Whittier KL, Hegarty DM, Aicher SA (2008) Periaqueductal gray neurons project to spinally projecting GABAergic neurons in the rostral ventromedial medulla. Pain 140:376-386. CrossRef Medline

Navratilova E, Atcherley CW, Porreca F (2015) Brain circuits encoding reward from pain relief. Trends Neurosci 38:741-750. CrossRef Medline

Nichols TE, Holmes AP (2002) Nonparametric permutation tests for functional neuroimaging: a primer with examples. Hum Brain Mapp 15:1-25. CrossRef Medline

O’Doherty JP, Dayan P, Friston K, Critchley H, Dolan RJ (2003) Temporal difference models and reward-related learning in the human brain. Neuron 38:329-337. CrossRef Medline

Padoa-Schioppa C, Assad JA (2006) Neurons in the orbitofrontal cortex encode economic value. Nature 441:223-226. CrossRef Medline

Palminteri S, Justo D, Jauffret C, Pavlicek B, Dauta A, Delmaire C, Czernecki V, Karachi C, Capelle L, Durr A, Pessiglione M (2012) Critical roles for anterior insula and dorsal striatum in punishment-based avoidance learning. Neuron 76:998-1009. CrossRef Medline

Pearce JM, Hall G (1980) A model for Pavlovian learning: variations in the effectiveness of conditioned but not of unconditioned stimuli. Psychol Rev 87:532-552. CrossRef Medline

Ploghaus A, Tracey I, Clare S, Gati JS, Rawlins JN, Matthews PM (2000) Learning about pain: the neural substrate of the prediction error for aversive events. Proc Natl Acad Sci U S A 97:9281-9286. CrossRef Medline

Ploghaus A, Narain C, Beckmann CF, Clare S, Bantick S, Wise R, Matthews PM, Rawlins JN, Tracey I (2001) Exacerbation of pain by anxiety is associated with activity in a hippocampal network. J Neurosci 21:98969903. CrossRef Medline

Porro CA, Baraldi P, Pagnoni G, Serafini M, Facchin P, Maieron M, Nichelli P (2002) Does anticipation of pain affect cortical nociceptive systems? J Neurosci 22:3206-3214. CrossRef Medline

Price DD (2000) Psychological and neural mechanisms of the affective dimension of pain. Science 288:1769-1772. CrossRef Medline

Rescorla RA, Wagner AR (1972) A theory of Pavlovian conditioning: Variations in the effectiveness of reinforcement and nonreinforcement. In: Classical conditioning ii: current research and theory (Black AH, Prokasy WF, eds), pp 64-99. New York: Appleton Crofts.

Rogers RD, Owen AM, Middleton HC, Williams EJ, Pickard JD, Sahakian BJ, Robbins TW (1999) Choosing between small, likely rewards and large, unlikely rewards activates inferior and orbital prefrontal cortex. J Neurosci 19:9029-9038. CrossRef Medline

Roy M, Shohamy D, Daw N, Jepma M, Wimmer GE, Wager TD (2014) Representation of aversive prediction errors in the human periaqueductal gray. Nat Neurosci 17:1607-1612. CrossRef Medline

Rudebeck PH, Murray EA (2014) The orbitofrontal oracle: cortical mechanisms for the prediction and evaluation of specific behavioral outcomes. Neuron 84:1143-1156. CrossRef Medline
Schenk LA, Sprenger C, Onat S, Colloca L, Büchel C (2017) Suppression of striatal prediction errors by the prefrontal cortex in placebo hypoalgesia. J Neurosci 37:9715-9723. CrossRef Medline

Schultz W (1998) Predictive reward signal of dopamine neurons. J Neurophysiol 80:1-27. CrossRef Medline

Schultz W (2016) Dopamine reward prediction-error signalling: a twocomponent response. Nat Rev Neurosci 17:183-195. CrossRef Medline

Schultz W, Dayan P, Montague PR (1997) A neural substrate of prediction and reward. Science 275:1593-1599. CrossRef Medline

Seidel EM, Pfabigan DM, Hahn A, Sladky R, Grahl A, Paul K, Kraus C, Küblböck M, Kranz GS, Hummer A, Lanzenberger R, Windischberger C, Lamm C (2015) Uncertainty during pain anticipation: the adaptive value of preparatory processes. Hum Brain Mapp 36:744-755. CrossRef Medline

Seymour B, O’Doherty JP, Dayan P, Koltzenburg M, Jones AK, Dolan RJ, Friston KJ, Frackowiak RS (2004) Temporal difference models describe higher-order learning in humans. Nature 429:664-667. CrossRef Medline

Seymour B, O’Doherty JP, Koltzenburg M, Wiech K, Frackowiak R, Friston K, Dolan R (2005) Opponent appetitive-aversive neural processes underlie predictive learning of pain relief. Nat Neurosci 8:1234-1240. CrossRef Medline

Seymour B, Daw N, Dayan P, Singer T, Dolan R (2007) Differential encoding of losses and gains in the human striatum. J Neurosci 27:4826-4831. CrossRef Medline

Sutton RS, Barto AG (1998) Reinforcement learning: an introduction. Cambridge, MA: MIT.

Tracey I, Mantyh PW (2007) The cerebral signature for pain perception and its modulation. Neuron 55:377-391. CrossRef Medline

Tseng MT, Chiang MC, Yazhuo K, Chao CC, Tseng WY, Hsieh ST (2013) Effect of aging on the cerebral processing of thermal pain in the human brain. Pain 154:2120-2129. CrossRef Medline

Tzourio-Mazoyer N, Landeau B, Papathanassiou D, Crivello F, Etard O, Delcroix N, Mazoyer B, Joliot M (2002) Automated anatomical labeling of activations in SPM using a macroscopic anatomical parcellation of the MNI MRI single-subject brain. Neuroimage 15:273-289. CrossRef Medline

Wiech K (2016) Deconstructing the sensation of pain: the influence of cognitive processes on pain perception. Science 354:584-587. CrossRef Medline

Wiech K, Lin CS, Brodersen KH, Bingel U, Ploner M, Tracey I (2010) Anterior insula integrates information about salience into perceptual decisions about pain. J Neurosci 30:16324-16331. CrossRef Medline

Winston JS, Vlaev I, Seymour B, Chater N, Dolan RJ (2014) Relative valuation of pain in human orbitofrontal cortex. J Neurosci 34:14526-14535. CrossRef Medline

Yacubian J, Gläscher J, Schroeder K, Sommer T, Braus DF, Büchel C (2006) Dissociable systems for gain- and loss-related value predictions and errors of prediction in the human brain. J Neurosci 26:9530-9537. CrossRef Medline

Yezierski RP (1988) Spinomesencephalic tract: projections from the lumbosacral spinal cord of the rat, cat, and monkey. J Comp Neurol 267:131146. CrossRef Medline

Yoshida W, Seymour B, Koltzenburg M, Dolan RJ (2013) Uncertainty increases pain: evidence for a novel mechanism of pain modulation involving the periaqueductal gray. J Neurosci 33:5638-5646. CrossRef Medline 